\title{
10. Therapie der akuten septischen Kardiomyopathie
}

\author{
K. Werdan
}

Die Herzinsuffizienz bei Sepsis/septischem Schock und Multiorganversagen - im folgenden als „akute septische Kardiomyopathie“ [26] bezeichnet - bietet im Vergleich zu nichtseptischen Formen der Herzinsuffizienz einige Besonderheiten - mit differentialtherapeutischen Konsequenzen. Die Therapie der eingeschränkten Herzfunktion darf nicht isoliert betrachtet werden: sie muß sich in ein Gesamtkonzept einordnen, das die zusätzlichen Störungen im Bereich der Makro- und Mikrozirkulation sowie auf Organebene mitberücksichtigt (vgl. Kap.3). Sie ist derzeit noch überwiegend symptomatisch; mit zunehmendem Verständnis der Ursachen der akuten septischen Kardiomyopathie zeichnen sich jedoch bereits auch Ansätze einer kausaleren Behandlungsweise ab. Obwohl nicht nur die systolische, sondern auch die diastolische Herzfunktion in der Sepsis beeinträchtigt ist (vgl. Kap.2), konzentriert sich derzeit unser Interesse noch weitgehend auf die Besserung der systolischen Funktionsstörung; eine Funktions-

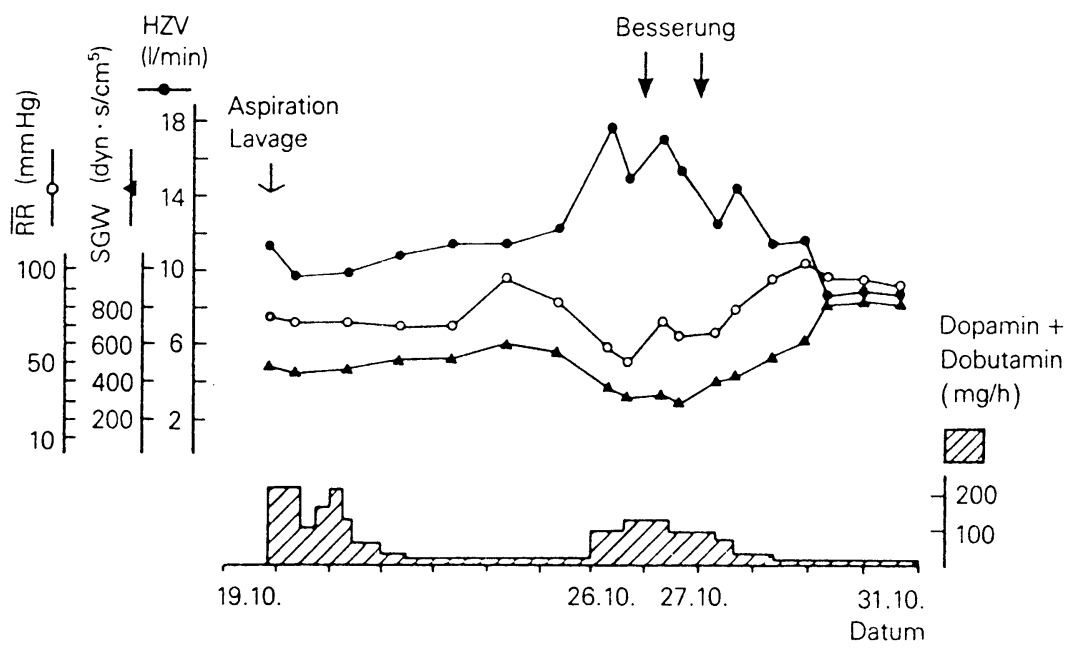

Abb. 10.1. Kasuistik: Herz-Kreislauf-Befunde bei einem 47jährigen Patienten mit Pseudomonassepsis nach Aspirationspneumonie. Aspirationspneumonie am 19.10. Nach initialer Befundbesserung kommt es bis 26.10. zu einer Befundverschlechterung mit katecholaminpflichtiger Schocksymptomatik. Ab 27.10. tritt eine anhaltende klinische Besserung ein. (Aus Werdan et al. [26]) 
einschränkung des rechten Ventrikels infolge pulmonaler Hypertonie bei ARDS und bestehende kardiale Vorerkrankungen erfordern zusätzliche differentialtherapeutische Überlegungen. Unsere Behandlungskonzepte wurden überwiegend bei Patienten mit primär septischem Krankheitsbild und Multiorganversagen erarbeitet. Über die Mechanismen der Herzinsuffizienz bei primär nichtseptischem Multiorganversagen - z. B. nach schwerem Trauma - ist dagegen sehr viel weniger bekannt.

\section{Besonderheiten der Herzinsuffizienz bei Sepsis/septischem Schock und Multiorganversagen: akute septische Kardiomyopathie}

Die Kasuistik von Abb. 10.1 verdeutlicht die typischen Herz-Kreislauf-Veränderungen, wie sie bei gramnegativem und grampositivem septischem Schock im Rahmen des Multiorganversagens gefunden werden: Der Blutdruckabfall infolge der Erniedrigung des systemischen Gefäßwiderstands bis auf etwa $30 \%$ der Norm

Abb. 10.2. Herzfunktionsparameter von 2 Patienten mit septischem Schock $(O, \square)$ in Abhängigkeit vom systemischen Gefäßwiderstand. Bei diesen beiden Patienten wurden während der Krankheitsverläufe mit klinischer Befundbesserung in einem Zeitraum von 20 Tagen (Patient 1 , O) bzw. von 8 Tagen (Patient 2, $\square$ ) mehrfach Herzfunktionsparameter mittels Swan-Ganz-Katheter ermittelt: Herzindex, linksventrikulärer Schlagarbeitsindex, Schlagvolumenindex. Die Werte wurden in Abhängigkeit von dem jeweils aktuellen systemischen Gefäßwiderstand aufgetragen. Zum Vergleich sind die Normalwerte Gesunder ( $\bullet$ ) aufgeführt. (Aus Niemer et al. [11], S. 367)

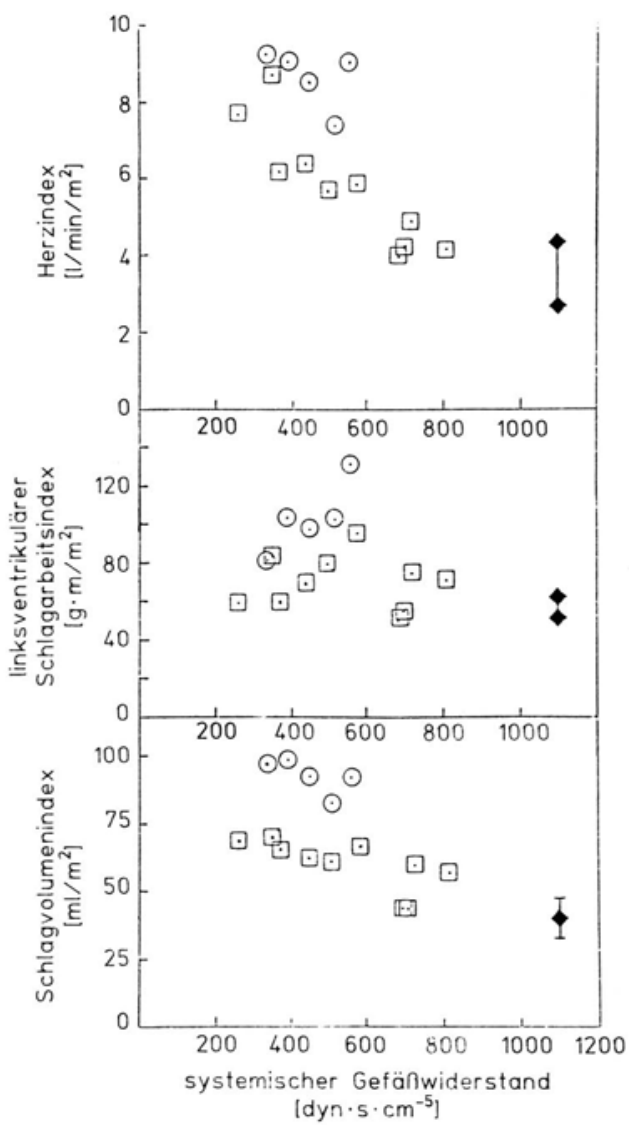


kann bis zu einem gewissen Grade durch einen Anstieg des Herzzeitvolumens kompensiert werden. Herzzeitvolumen/Herzindex, Schlagvolumenindex und linksventrikulärer Schlagarbeitsindex steigen dabei um so mehr an, je stärker der systemische Gefäßwiderstand abfällt (Abb. 10.2). Fehlt eine relevante kardiale Vorschädigung, so liegen die gemessenen Herzfunktionsparameter dabei auch beträchtlich höher als die gesunder Probanden mit einem „normalen“ systemischen Gefäßwiderstand um $1100 \mathrm{dyn} \cdot \mathrm{cm}^{-5} \cdot \mathrm{s}$ (Abb. 10.2).

Eine so weitgehende Kompensation - d. h. ein Anstieg des Herzzeitvolumens auf das Zwei- bis Dreifache der Norm - wird allerdings im septischen Schock nur selten beobachtet, v. a. nicht bei protrahierten Verläufen: die Pumpfunktionsparameter des Herzens sind dabei zwar im Vergleich zu gesunden Probanden mit „normalem“ systemischen Gefäßwiderstand meist nicht erniedrigt oder sogar leicht erhöht; berücksichtigt man jedoch die inverse Korrelation mit dem systemischen Gefäßwiderstand (Abb. 10.2; Kap. 2: Abb. 2.5), so wird die eingeschränkte Pumpleistung des Herzens bei vielen Patienten bereits in der hyperdynamen Phase des septischen Schocks und sogar bereits bei septischen Patienten mit noch normalem Blutdruck [19] rasch evident. Bezieht man weiterhin auch noch die in der Sepsis vorhandene $\mathrm{O}_{2}$-Verwertungsstörung im Gewebe mit verminderter $\mathrm{O}_{2}$-Extraktion (Kap.9) und erhöhtem Skelettmuskel- $\mathrm{O}_{2}$-Partialdruck (Abb. 10.3.) in die Überlegungen mit ein, so wird klar, daß die Behandlung

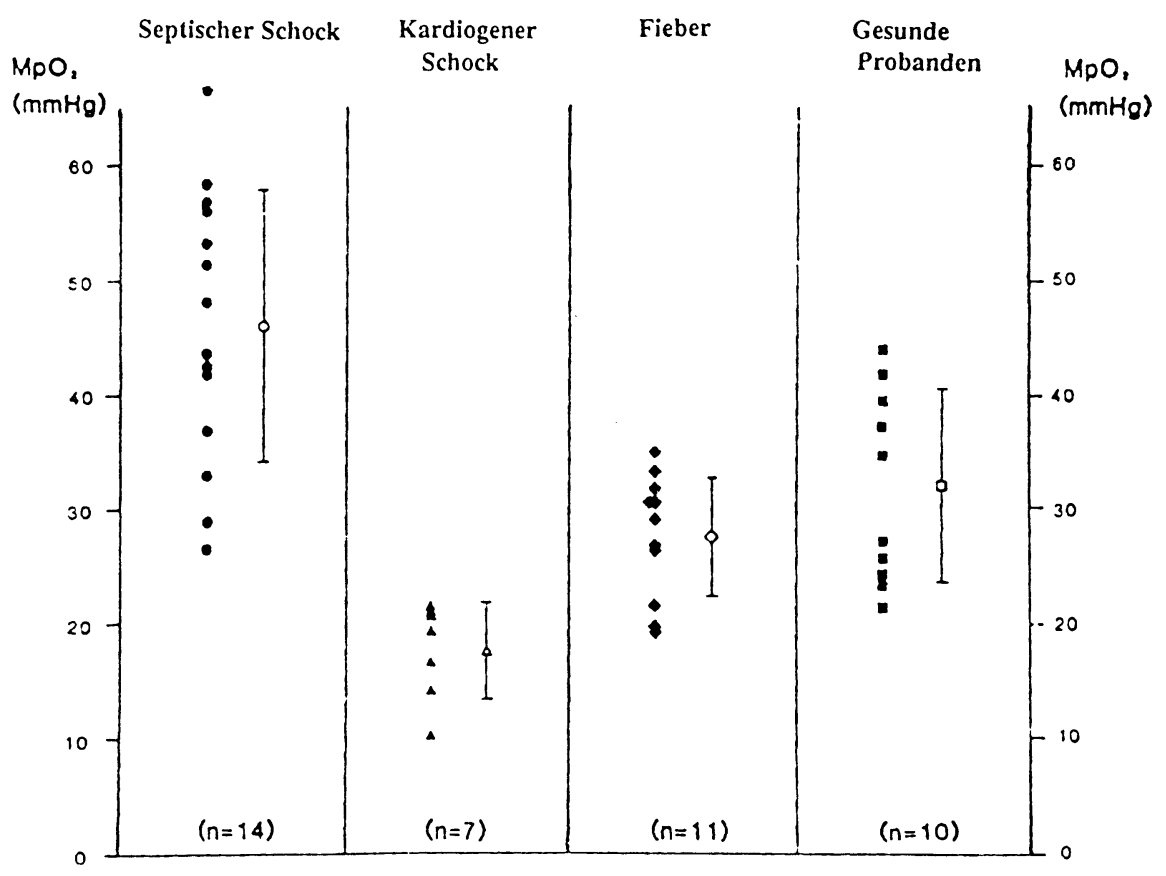

Abb. 10.3. Skelettmuskel(Musculus-biceps-brachii)- $\mathrm{O}_{2}$-Partialdruckmessungen bei Patienten mit Sepsis, kardialem Pumpversagen, Fieber und bei gesunden Probanden. (Aus Werdan et al. [26]) 
der akuten Herzinsuffizienz in der Sepsis eine eigenständige Betrachtungweise erfordert, die sich nicht ausschließlich an der Behandlung der nichtseptisch bedingten akuten Herzinsuffizienz orientieren kann.

Bei jedem Patienten mit drohender oder manifester Sepsis bzw. mit drohendem oder manifestem Multiorganversagen sollte die akute septische Kardiomyopathie in dreierlei Weise zum frühestmöglichen Zeitpunkt charakterisiert werden:

a) Schweregrad der akuten septischen Kardiomyopathie: dies gelingt durch Beurteilung der Herzfunktionsparameter in Abhängigkeit vom systemischen Gefäßwiderstand (Abb. 10.2) mittels Swan-Ganz-Katheter; echokardiographisch wird der Schweregrad dagegen häufig unterschätzt.

b) Funktionelle Relevanz der akuten septischen Kardiomyopathie: diese ist um so bedeutungsvoller, je ausgeprägter die Organperfusion und damit die Organfunktion in der Sepsis und im Multiorganversagen bereits beeinträchtigt ist: SIRS/Sepsis - sepsisinduzierte Hypotonie/MODS/schwere Sepsis - septischer Schock - refraktärer septischer Schock (zur Terminologie s. unten);

c) Vorliegen kardialer Begleiterkrankungen: sie können sich zusätzlich auf die Herzfunktion ungünstig auswirken (s. S. 195).

\section{Terminologie}

Mit Ausnahme der letzten beiden Begriffe: in Anlehnung an The ACCP/SCCM Consensus Conference Committee (1992) Definitions for sepsis and organ failure and guidelines for the use of innovative therapies in sepsis. Chest 101:1644-1655.

\section{Infektion}

Entzündliche Gewebereaktion auf Mikroorganismen oder Invasion von Mikroorganismen in normalerweise steriles Gewebe.

\section{Bakteriämie}

Vorhandensein vitaler Bakterien im Blut.

\section{SIRS (,systemic inflammatory response syndrome“)}

Systemisch-entzündliche Reaktion auf verschiedene schwere klinische Insulte, charakterisiert durch 2 oder mehr der folgenden Symptome:

1) Körpertemperatur $>38^{\circ} \mathrm{C}$ oder $<36^{\circ} \mathrm{C}$,

2) Herzfrequenz $>90 / \mathrm{min}$,

3) Atemfrequenz $>20 / \mathrm{min}$ oder $\mathrm{p}_{\mathrm{a}} \mathrm{CO}_{2}<32 \mathrm{~mm} \mathrm{Hg}$,

4) Leukozyten $>12000 / \mathrm{mm}^{3}$ oder $<4000 / \mathrm{mm}^{3}$, oder $>10 \%$ unreife (stabförmige) Formen.

Sepsis

Systemische Reaktion auf eine Infektion, charakterisiert durch 2 oder mehr der folgenden, durch die Infektion hervorgerufenen Symptome:

1) Körpertemperatur $>38^{\circ} \mathrm{C}$ oder $<36^{\circ} \mathrm{C}$,

2) Herzfrequenz $>90 / \mathrm{min}$,

3) Atemfrequenz $>20 /$ min oder $\mathrm{p}_{\mathrm{a}} \mathrm{CO}_{2}<32 \mathrm{~mm} \mathrm{Hg}$,

4) Leukozyten $>12000 / \mathrm{mm}^{3}$ oder $<4000 / \mathrm{mm}^{3}$, oder $>10 \%$ unreife (stabförmige) Formen. 


\section{Schwere Sepsis}

Sepsis, assoziiert mit Organdysfunktion, Minderperfusion oder Hypotonie. Minderdurchblutung und Durchblutungsstörungen können beinhalten, sind aber nicht beschränkt auf: Laktatazidose, Oligurie oder eine akute Änderung der Bewußtseinslage.

MODS (,multiple organ dysfunction syndrome“)

Vorhandensein einer dermaßen geänderten Organfunktion bei Akutkranken, daß die Homöostase ohne Intervention nicht mehr aufrechterhalten werden kann.

\section{Sepsisinduzierte Hypotonie}

Systolischer Blutdruck $<90 \mathrm{~mm} \mathrm{Hg}$ oder eine Reduktion um $\geq 40 \mathrm{~mm} \mathrm{Hg}$ vom Ausgangswert - bei Fehlen anderer Hypotonieursachen.

\section{Septischer Schock}

Sepsisinduzierter Schock mit Hypotonie trotz adäquater Volumensubstitution, einhergehend mit Durchblutungsstörungen; letztere können beinhalten, sind aber nicht beschränkt auf: Laktatazidose, Oligurie oder eine akute Änderung der Bewußtseinslage. Die Behandlung mit inotropen oder vasokonstriktorischen Substanzen kann zur Maskierung der Hypotonie zum Zeitpunkt der Feststellung von Durchblutungsstörungen führen.

\section{Refraktärer septischer Schock}

Septischer Schock ohne rasches Ansprechen auf Volumengabe (z. B. $500 \mathrm{ml} \mathrm{NaCl}$ in $30 \mathrm{~min}$ ) und Vasopressoren (z. B. Dopamin $>10 \mu \mathrm{g} / \mathrm{kg} \mathrm{KG} / \mathrm{min}$ ).

\section{Akute septische Kardiomyopathie}

Myokardschädigung im Rahmen einer Sepsis mit der Folge einer im Verhältnis zum systemischen Gefäßwiderstand verminderten Pumpfunktion des Herzens.

\section{Behandlungsziele bei akuter septischer Kardiomyopathie}

Voraussetzung für eine wirksame Therapie ist eine möglichst frühzeitige Diagnose und Schweregradeinschätzung der septischen Herz-Kreislauf-Insuffizienz; intraarterielle Druckregistrierung und ein Pulmonaliskatheter mit Thermodilutionsmessung erlauben die Messung und Berechnung aller zur Überwachung solcher kritisch Kranker notwendigen hämodynamischen Daten; nach Parrillo [12] sollten alle Patienten mit der Verdachtsdiagnose eines septischen Schocks mit diesem hämodynamischen Monitoring überwacht werden; die gleiche Empfehlung findet sich für Patienten mit septischem Schock bei Niemer et al. ([11, S. 1592), und sie wird auch für kritisch Kranke mit Sepsis von den zuständigen amerikanischen Ärztevereinigungen (Zitat 17 in [25]) gegeben. Zur Beurteilung des Blutdrucks sind der arterielle Mitteldruck (s. Übersicht unten und Tabelle 10.1) und in der Regel auch der systolische Blutdruck (vgl. „Terminologie“ S. 167/168) geeignet; allerdings kann bereits bei Vorliegen eines niedrigen diastolischen Drucks trotz eines systolischen Blutdrucks von $90 \mathrm{~mm} \mathrm{Hg}$ eine Organminderperfusion vorliegen [12]. 


\section{Zielkriterien bei der Behandlung der akuten septischen Kardiomyopathie und des septischen Schocks}

\section{Blutdruck}

Mittlerer arterieller Blutdruck $\geq 60 \mathrm{~mm} \mathrm{Hg}$.

\section{Organdurchblutung}

Die Durchblutung - z. B. von Nieren, Leber, ZNS und Lungen - wird als adäquat angesehen, wenn die Organfunktion nicht eingeschränkt ist. Eine Urinausscheidung von $>20 \mathrm{ml} / \mathrm{h}$ zeigt indirekt eine ausreichende Nierendurchblutung an [12]. Verhinderung eines Anstiegs des Blutlaktats.

Parameter des $\mathrm{O}_{2}$-Stoffwechsels und der Gewebeoxygenierung: s. Kap. 9.

Quantifizierung des Schweregrades der septisch bedingten Gefäßschädigung mit Vasodilatation

Systemischer Gefäßwiderstand: Abweichung vom Sollwert $\left(1100 \pm 200 \mathrm{dyn} \cdot \mathrm{cm}^{-5} \cdot \mathrm{s} ; \mathrm{s}\right.$. Abb. 10.1).

\section{Quantifizierung des Schweregrades der akuten septischen Kardiomyopathie}

Sollwertabweichungen von Pumpfunktionsparametern (z. B. Herzeitvolumen/ Herzindex, Schlagvolumenindex, rechts- und linksventrikulärer Schlagarbeitsindex; rechts- und linksventrikuläre Auswurffraktion), betrachtet in Relation zum aktuellen systemischen Gefäßwiderstand (s. Abb. 2.5, 10.2).

Siehe dazu auch Tabelle 10.1.

Tabelle 10.1. Richtwerte hämodynamischer Parameter bei Patienten mit drohender oder manifester Sepsis und Multiorganversagen. (Aus Conrad et al. [2])

\begin{tabular}{|c|c|c|c|}
\hline Parameter & $\begin{array}{l}\text { Normal- } \\
\text { wert }\end{array}$ & $\begin{array}{l}\text { Richt- } \\
\text { wert }\end{array}$ & Einheit \\
\hline Herzfrequenz & $70-90$ & $<120$ & $\min 1$ \\
\hline Mittlerer arterieller Blutdruck & $80-100$ & $>70$ & $\mathrm{~mm} \mathrm{Hg}$ \\
\hline Mittlerer Pulmonalarteriendruck & $12-16$ & $<20$ & $\mathrm{mmHg}$ \\
\hline Pulmonalkapillardruck & $8-12$ & $12-16$ & $\mathrm{mmHg}$ \\
\hline Rechtsatrialer Druck & $5-8$ & $<8$ & $\mathrm{mmHg}$ \\
\hline Herzindex & $3,5-4,5$ & $>4,5$ & $1 \cdot \min \cdot \mathrm{m}^{2}$ \\
\hline Schlagvolumenindex & $35-45$ & $>45$ & $\mathrm{ml} \cdot \mathrm{m}^{-2}$ \\
\hline Linksventrikulärer Schlagarbeitsindex & $45-65$ & $>45$ & $\mathrm{~g} \cdot \mathrm{m} \cdot \mathrm{m}^{2}$ \\
\hline Rechtsventrikulärer Schlagarbeitsindex & $4-8$ & $>10$ & $\mathrm{~g} \cdot \mathrm{m} \cdot \mathrm{m}^{2}$ \\
\hline Systemischer Gefäßwiderstand & $1800-2400$ & $1200-2000$ & $\mathrm{dyn} \cdot \mathrm{s} \cdot \mathrm{cm}^{5} \cdot \mathrm{m}^{2}$ \\
\hline Pulmonaler Gefäßwiderstand & $50-200$ & $<250$ & $\operatorname{dyn} \cdot \mathrm{s} \cdot \mathrm{cm}^{5} \cdot \mathrm{m}^{2}$ \\
\hline Arterieller $\mathrm{O}_{2}$-Partialdruck & $90-95$ & $>60$ & $\mathrm{mmHg}$ \\
\hline Arterielle $\mathrm{O}_{2}$-Sättigung & $95-100$ & $>90$ & $\%$ \\
\hline Gemischvenöser $\mathrm{O}_{2}$-Partialdruck & $35-50$ & $>35$ & $\mathrm{~mm} \mathrm{Hg}$ \\
\hline Gemischvenöse $\mathrm{O}_{2}$-Sättigung & $70-75$ & $>60$ & $\%$ \\
\hline $\mathrm{O}_{2}$-Angebot & $550-650$ & $>650$ & $\mathrm{ml} \cdot \min ^{1} \cdot \mathrm{m}^{2}$ \\
\hline $\mathrm{O}_{2}$-Verbrauch & $110-150$ & $>170$ & $\mathrm{ml} \cdot \min ^{1} \cdot \mathrm{m}^{2}$ \\
\hline $\mathrm{O}_{2}$-Extraktionsverhältnis & $25-30$ & $<30$ & $\%$ \\
\hline Pulmonale Shuntfraktion & $3-5$ & $<5$ & $\%$ \\
\hline
\end{tabular}


Die Vasodilatation läßt sich an der Erniedrigung des systemischen Gefäßwiderstands und die akute septische Kardiomyopathie an der - in Relation zum systemischen Gefäßwiderstand - verminderten Herzfunktion (z. B. Herzindex, Schlagvolumenindex, linksventrikulärer Schlagarbeitsindex; Abb. 2.5, Abb. 10.2) erkennen. Das klinische Erscheinungsbild des septischen Schocks stellt bereits ein weit fortgeschrittenes Krankheitsstadium dar! Der septische Schock ist in der Regel hyperdynam (Herzindex $>5,5 \mathrm{l} / \mathrm{min} \cdot \mathrm{m}^{2}$; systemischer Gefäßwiderstand $\leq 600$ dyn $\cdot \mathrm{s} \cdot \mathrm{cm}^{-5}$ ) und nur in der Spätphase oder bei nicht ausreichender Volumensubstitution hypodynam ( $<2,5 ; \geq 1200 ;$ Klassifikation nach [11], S. 1553 und 1581-1584).

Eine Einschränkung der links- und rechtsventrikulären Pumpfunktion findet sich nicht erst im septischen Schock, sondern bereits bei normotensiven Sepsispatienten [18]. Die Therapie dieser akuten septischen Kardiomyopathie beginnt somit nicht erst beim manifesten septischen Schock, sondern spätestens bei der sepsisbedingten Hypotonie oder - trotz normalen Blutdrucks - bei Vorliegen einer Organminderperfusion! Je schneller die Diagnose gestellt wird und je rascher und konsequenter die kurativen Therapiemaßnahmen eingeleitet werden, desto besser ist die Prognose dieser Patienten ([11], S. 1585).

Die akute septische Kardiomyopathie ist vorwiegend durch eine primär myokardiale systolische und diastolische Funktionsstörung des linken und rechten Ventrikels charakterisiert (s. Kap. 2), während eine verminderte $\mathrm{O}_{2}$-Versorgung des Herzens infolge von Koronarstenosen nur bei Patienten mit zusätzlich bestehender koronarer Herzkrankheit oder bei Hypotonie zu erwarten ist. Eine pulmonale Hypertonie im Rahmen eines ARDS kann darüber hinaus zu einer zusätzlichen Belastung des rechten Ventrikels führen, verbunden mit einer mechanischen Beeinträchtigung des linken Ventrikels durch ein Kammerseptumshift nach links.

Aus dem Gesagten wird ersichtlich, daß neben der Besserung einer pulmonalen Hypertonie bei ARDS (s. S. 194-195; s. auch Kap. 11) v. a. die Besserung der systolischen und diastolischen Funktionsstörung des linken Ventrikels therapeutische Zielkriterien sind, um letztendlich die Organperfusion in der Sepsis zu optimieren. Hierfür stehen - nach Ausschöpfung der gesicherten Kausaltherapie mit radikaler Herdsanierung und adäquater Antibiotikatherapie - symptomatische Maßnahmen und weitere kausale, in ihrer Wirksamkeit aber noch wenig bis nicht gesicherte Behandlungsversuche zur Verfügung.

\section{Behandlung der akuten septischen Kardiomyopathie und des septischen Schocks}

\section{Bestätigung der korrekten Diagnose}

A) Differentialdiagnose des Schocks: Rechtsherzkatheterisierung

B) Intraarterielle Druckmessung: Ungenauigkeit der Blutdruckmessung mit Manschette im Schock

C)* Quantifizierung von Schweregrad und funktioneller Relevanz der akuten septischen Kardiomyopathie (s. S. 165-168) 
II. Sicherung des sofortigen Erkennens und der raschen Behandlung von Blutdruckänderungen

Blutdruckinstabilität im septischen Schock

III. Elimination von Ursachen, die zur Myokarddepression und Stoffwechselentgleisung führen
A) Korrektur einer Hypoxie
B) Korrektur einer Azidose
C) Korrektur von Hypophosphatämie und Hypokalzämie
D) Korrektur von sonstigen Elektrolytstörungen

IV. Hämodynamisches Monitoring zur Therapieoptimierung: Volumensubstitution

A) Optimierung der Vorlast (s. S. 172-174, 185)

1) Pulmonalkapillardruck

2) rechtsatrialer Druck

3) Vermeidung eines Lungenödems

B) Therapie mit inotropen und vasokonstriktorischen Substanzen (s. S. 174-185)

1) Optimierung des Herzzeitvolumens/Herzindex

2) nach Erzielen des maximalen Herzzeitvolumens/Herzindex: pharmakologische Blutdrucksteigerung mit Nachlasterhöhung angezeigt

3) Notwendigkeit einer exakten Bilanzierung der intravenösen Volumensubstitution

C) Optimierung der Organdurchblutung (s. auch Kap. 8)

1) Oligurie

a) Maximierung des Herzzeitvolumens mittels Volumensubstitution

b) Dopamin, Noradrenalin, andere Vasokonstriktoren

c) Vasodilatatoren

2) pulmonale Shunts (s. auch Kap. 11)

3) Hirndurchblutung

4) Leberdurchblutung

V. Rhythmusmonitoring (s. S. 186)

A) Antiarrhythmika

B) elektrische Kardioversion

VI.* Optimierung der Atmung/Beatmung und Reduktion der Herzarbeit (s. S. 186-187)

A) Optimierung der Oxygenierung (Kontrollparameter: $\mathrm{p}_{\mathrm{a}} \mathrm{O}_{2} ; \mathrm{S}_{\mathrm{a}} \mathrm{O}_{2}$ )

B) Cave: erhöhte Atemarbeit

C) Belastung des Herzens durch PEEP, CMV, IMV

D) Anxiolyse, Analgesie, Fiebersenkung

E) Cave: myokarddepressive Medikation: Anästhetika, Barbiturate, Meperiden, Kombination von Opiaten und Benzodiazepinen

VI. Fokussanierung, Antibiotika (s. auch Kap. 4)

VII. Kausale Therapieansätze (s. S. 187-194)

* Mit Ausnahme von I. C), VI. und VII. in Anlehnung an Parrillo [12]. 
Anstelle der komplexen pathophysiologischen Definition des septischen Schocks wird als praktikabler Parameter zur Therapieentscheidung die Höhe des arteriellen Blutdrucks herangezogen: Zur Sicherung einer ausreichenden Koronar- und Zerebralperfusion sollte der arterielle Mitteldruck $60 \mathrm{~mm} \mathrm{Hg}$ und der systolische Blutdruck $90 \mathrm{~mm} \mathrm{Hg}$ nicht unterschreiten [12].

Diesem häufig praktizierten Vorgehen stehen Vorschläge gegenüber, die sich mehr an therapeutischen Zielkriterien (s. S. 169) orientieren: an der Sicherung einer ausreichenden Organdurchblutung und $\mathrm{O}_{2}$-Versorgung sowie der Verringerung des Schweregrades von septisch bedingter Gefäßschädigung und akuter septischer Kardiomyopathie. Entsprechende Richtwertvorschläge sind in Tabelle 10.1 aufgeführt. Inwieweit dadurch eine Prognoseverbesserung zu erzielen ist, bleibt Gegenstand der Diskussion.

\section{Symptomatische Therapie der akuten septischen Kardiomyopathie}

Die symptomatische Therapie beinhaltet als erstes die Volumensubstitution und danach die Gabe von positiv inotropen und vasoaktiven Substanzen.

\section{Volumensubstitution}

Die sepsisbedingte Gefäßschädigung mit Abstrom großer Flüssigkeitsmengen ins Interstitium birgt die Gefahr einer intravasalen Hypovolämie in sich, mit weiterer Verschlechterung der Zirkulation durch Blutdruckabfall und Abnahme des Herzzeitvolumens. Frühzeitige Volumenzufuhr ist unbestritten die wichtigste therapeutische Maßnahme, um rasch adäquate ventrikuläre Füllungsdrücke wiederherzustellen und die Herzfunktion zu bessern (Abb. 10.4; [17]); die dazu notwendigen, teils großen Flüssigkeitsmengen lassen es dringend ratsam erscheinen, diese Volumensubstitution bei Patienten mit septischem Schock unter regelmäßigem hämodynamischem Monitoring mittels Swan-Ganz-Katheter vorzunehmen. Die individuelle Einstellung des rechtsatrialen Drucks auf mindestens $10-12 \mathrm{~mm} \mathrm{Hg}$ und des Pulmonalkapillardrucks auf etwa $12-16-18 \mathrm{~mm} \mathrm{Hg}$ ([11]; 12], S. 1585) erscheint hierbei ratsam, wobei aufgrund der erhöhten Dehnbarkeit des Herzens in der Sepsis relativ große Flüssigkeitsmengen erforderlich sind. Die gleichzeitige Bestimmung des Herzzeitvolumens bei verschiedenen Rechtsherzkatheterdruckwerten trägt zur Optimierung dieses Vorgehens bei und läßt rechtzeitig eine volumenbedingte Drucküberlastung mit Lungenstauung und Abnahme des Herzzeitvolumens erkennen. Bei koronarkranken Septikern mit „starrem“ ischämischem Ventrikel ist allerdings bei der Volumensubstitution Vorsicht geboten ([18]; [20], S. 108-123).

Dieser großzügigen Volumensubstitution stehen manche Therapeuten aus Sorge vor peripherer und pulmonaler Ödembildung mit Einschränkung der Gewebeoxygenierung und der Gefahr der Zunahme pulmonaler Shunts eher zurückhaltend gegenüber: sie substituieren einen vorbestehenden Volumenmangel nur bis zum Erreichen von normalen oder nur gering erhöhten Füllungs- 


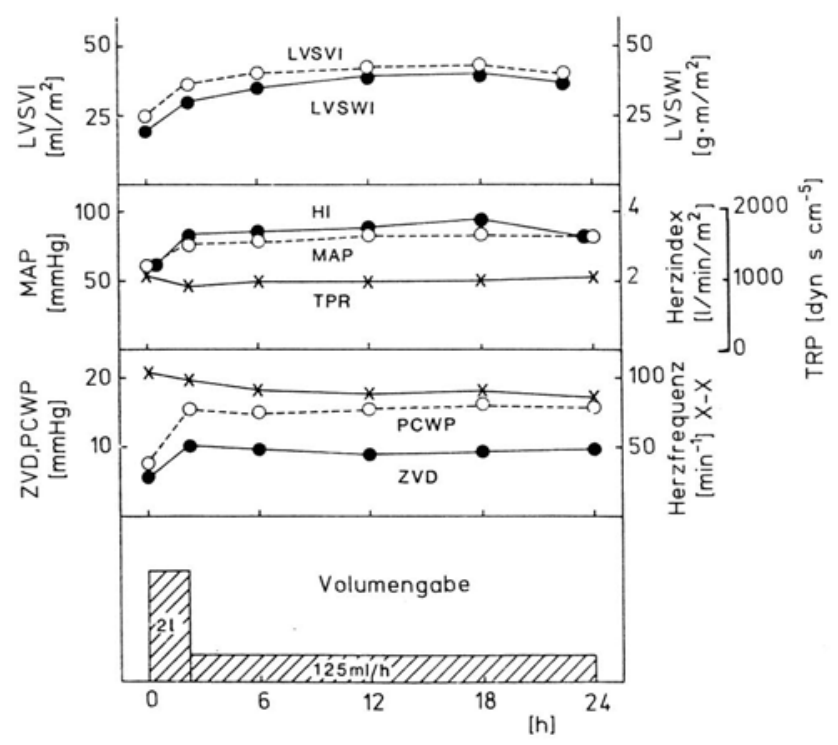

Abb. 10.4. Symptomatische Therapie des septischen Schocks: Volumensubstitution $(\mathrm{n}=18)$. Herz-Kreislauf-Veränderungen nach i.v.-Akutinfusion von 21 Flüssigkeit über $2 \mathrm{~h}$, anschließend $125 \mathrm{ml} / \mathrm{h}$ in den folgenden $22 \mathrm{~h}(Z V D$ zentraler Venendruck; $P C W P$ Pulmonalkapillardruck; $M A P$ mittlerer arterieller Druck; TPR systemischer Gefäßwiderstand; HI Herzindex; LVSWI linksventrikulärer Schlagarbeitsindex; LVSVI linksventrikulärer Schlagvolumenindex. (Nach Rackow et al. [17])

drücken und setzen anschließend vasokonstriktorische Katecholamine ein ([13], S. 89-93).

Ob kolloide oder kristalloide Lösungen zur Volumensubstitution bei septischen Patienten besser geeignet sind, wird seit langem kontrovers diskutiert; die Art der hierzu verwendeten Lösungen scheint allerdings nur eine untergeordnete Rolle zu spielen; Unterschiede bezüglich Morbidität und Mortalität konnten für verschiedene Flüssigkeitsregimes bisher nicht eindeutig gezeigt werden ([20], S. 252-260). Kristalloide Lösungen sind kostengünstig, leicht zu lagern, steigern ausreichend die Diurese und können zusätzlich extravasale Flüssigkeitsverluste bei Dehydratationszuständen ersetzen; nachteilig sind das Auftreten peripherer Ödeme und die relativ kurze hämodynamische Wirksamkeit. Kolloide Lösungen (Albumin, Hydroxyäthylstärke, Dextran 70) dagegen verbleiben zunächst vorwiegend im Intravasalraum und stellen dort den plasmaonkotischen Druck wieder her. Sie führen demzufolge weniger zu peripheren Ödemen, und es reichen geringere Volumina zur Substitution aus. Nachteile sind neben den hohen Kosten die bekannten, substanzeigenen Nebenwirkungen (Dextrane: allergische Reaktionen, Gerinnungsstörungen, Begünstigung eines akuten Nierenversagens; Hydroxyäthylstärke: Blutungen, weniger relevant als bei Dextranen; Albumin in großen Mengen: Senkung des ionisierten Plasmakalziums). Daß kolloidale Lösungen v. a. bei der Sepsis mit erhöhter Kapillarpermeabilität das Auftreten eines Lungenödems fördern, ist viel diskutiert, aber bisher nicht gesichert worden. 
Bei einem Hkt von weniger als $30 \%$ empfiehlt sich der Einsatz von Erythrozytenkonzentraten, und bei einem Serumalbumin von weniger als $2 \mathrm{~g} / \mathrm{dl}$ die Gabe von Albumin [12]. Es ist jedoch nicht sinnvoll, eine Hypalbuminämie als Folge eines kapillären Lecks - wie bei der Sepsis - mit Albumin vollständig auszugleichen, da dieses mit einer Halbwertzeit von 1-6h aus dem Intravasal- in den Extravasalraum abwandert.

Auch Infusionen aus Glukose, Insulin und Kalium werden beim septischen Schock eingesetzt ([20], S. 252-260). Der Stellenwert weiterer hypertoner Lösungen und insbesondere der hypertoner Kochsalzkolloidlösungen wird derzeit in experimentellen und klinischen Studien untersucht ([13], S. 104-118; [20], S. 252260 ; [23], S. 186-196).

\section{Katecholamine (Weiterführende Literatur in [25])}

\section{Grundlagen}

Die Therapie mit Katecholaminen und Sympathomimetika (im weiteren als Katecholamine bezeichnet) bei Patienten mit Sepsis/septischem Schock und Multiorganversagen hat zum Ziel, die Herz-Kreislauf-Schädigung zu kompensieren und damit die Durchblutung und die $\mathrm{O}_{2}$-Versorgung der Vitalorgane sicherzustellen (s. Kap. 9). Es handelt sich dabei um eine symptomatische, nicht um eine kausale Therapie.

Katecholamine entfalten ihre physiologischen und pharmakologischen Wirkungen durch Besetzung und Stimulation von Rezeptoren: am Herzen vorwiegend $\beta_{1}$-Adrenozeptoren und an den Gefäßen $\alpha_{1}$ - und $\beta_{2}$-Adrenozeptoren sowie Dopamin (DA-1 und DA-2)-Rezeptoren.

\section{Adrenozeptoren und Dopaminrezeptoren des Herz-Kreislauf-Systems}

Herz

$\beta_{1}$-Adrenozeptoren:

positiv inotrop und chronotrop

$\beta_{2}$ - und $\alpha_{1}$-Adrenozeptoren:

positiv inotrop (untergeordnete Bedeutung)

\section{Gefäße}

$\alpha_{1}$-Adrenozeptoren:

Vasokonstriktion (Arterien und Venen)

$\beta_{2}$-Adrenozeptoren:

Vasodilatation (Arterien)

Dopamin (DA)-Rezeptoren:

DA-1 (postsynaptisch): Vasodilatation (vorwiegend Nieren- und Mesenterialgefäße)

DA-2 (präsynaptisch): „passive Vasodilatation“ durch Hemmung der Noradrenalinfreisetzung 
Tabelle 10.2. Herz- und Gefäßwirkungen von Sympathomimetika. (Mod. nach Parrillo [12])

\begin{tabular}{|c|c|c|c|c|c|}
\hline \multirow{3}{*}{$\begin{array}{l}\text { Substanz } \\
\text { Dosierung }\end{array}$} & \multicolumn{2}{|c|}{ Herz-Wirkung } & \multicolumn{3}{|c|}{ Gefäß-Wirkung } \\
\hline & \multicolumn{2}{|c|}{$\beta_{1}$} & \multirow{2}{*}{$\begin{array}{l}\alpha \\
\text { Vasokon- } \\
\text { striktion }\end{array}$} & \multirow{2}{*}{$\begin{array}{l}\beta_{2} \\
\text { Vaso- } \\
\text { dilatation }\end{array}$} & \multirow[b]{2}{*}{$\begin{array}{l}\text { Dopamin- } \\
\text { erg }\end{array}$} \\
\hline & $\begin{array}{l}\text { Herz- } \\
\text { frequenz }\end{array}$ & $\begin{array}{l}\text { Kontrak- } \\
\text { tilität }\end{array}$ & & & \\
\hline $\begin{array}{l}\text { Dopamin } \\
1-10 \mu \mathrm{g} / \mathrm{kg} / \mathrm{min} \\
>10 \mu \mathrm{g} / \mathrm{kg} / \mathrm{min}\end{array}$ & $\begin{array}{l}2+ \\
2+\end{array}$ & $\begin{array}{l}2+ \\
2+\end{array}$ & $\begin{array}{l}0 \\
2-3+\end{array}$ & $\begin{array}{l}2+ \\
0\end{array}$ & $\begin{array}{l}4+ \\
0\end{array}$ \\
\hline $\begin{array}{l}\text { Dobutamin } \\
1-10 \mu \mathrm{g} / \mathrm{kg} / \mathrm{min}\end{array}$ & $1+$ & $4+$ & $1+$ & $2+$ & 0 \\
\hline $\begin{array}{l}\text { Noradrenalin } \\
2-8 \mu \mathrm{g} / \mathrm{min}\end{array}$ & $2+$ & $2+$ & $4+$ & 0 & 0 \\
\hline $\begin{array}{l}\text { Adrenalin } \\
1-8 \mu \mathrm{g} / \mathrm{min}\end{array}$ & $4+$ & $4+$ & $4+$ & $3+$ & 0 \\
\hline $\begin{array}{l}\text { Isoproterenol } \\
1-4 \mu \mathrm{g} / \mathrm{min}\end{array}$ & $4+$ & $4+$ & 0 & $4+$ & 0 \\
\hline $\begin{array}{l}\text { Phenylephrin } \\
20-200 \mu \mathrm{g} / \mathrm{min}\end{array}$ & 0 & 0 & $4+$ & 0 & 0 \\
\hline
\end{tabular}

Die Differentialtherapie mit Katecholaminen wird primär nicht durch unterschiedliche pharmakokinetische Eigenschaften bestimmt, sondern durch die unterschiedlichen Affinitäten der einzelnen Substanzen zu diesen verschiedenen Rezeptorarten (Tabelle 10.2). Dopamin und Dobutamin sind dabei in der Regel „Katecholamine der ersten Wahl“:

Dopamin in niedriger Dosierung stimuliert die Dopaminrezeptoren und wirkt dadurch v. a. auf die Nieren- und Mesenterialgefäße vasodilatierend (Steigerung der Nierenperfusion). Die gleichzeitige Stimulation der $\beta_{1}$-Adrenozeptoren ist für die positiv-inotrope und positiv-chronotrope Wirkung dieser Substanz verantwortlich. Bei hohen Dopaminkonzentrationen dominiert die vasokonstringierende $\alpha$-Adrenozeptorwirkung über die vasodilatierende dopaminerge Wirkung mit dem Nettoeffekt einer Vasokonstriktion auch der Nierenarterien.

Das für den klinischen Einsatz verfügbare Dobutamin ist ein Razemat aus 2 Enantiomeren; das (-)-Isomer bewirkt überwiegend eine $\alpha$-Adrenozeptorstimulation, das (+)-Isomer überwiegend eine $\beta_{1}$ - und auch eine $\beta_{2}$-Adrenozeptorstimulation. Der Nettoeffekt ist eine ausgeprägte positiv-inotrope Wirkung - bei nur geringer Frequenzbeeinflussung - und eine geringe Senkung des Gefäßwiderstands, bei weitgehend unverändertem Blutdruck.

Beim Noradrenalin steht neben der positiv-inotropen $\beta_{1}$-Adrenozeptorwirkung v. a. die Vasokonstriktion durch $\alpha$-Adrenozeptorstimulation im Vordergrund; sie ist verantwortlich für den ausgeprägten Blutdruckanstieg. Die Bindungsaffinität zu den $\beta_{2}$-Adrenozeptoren ist wesentlich geringer als die zu den $\beta_{1^{-}}$ Adrenozeptoren, so daß eine vasodilatierende Wirkung fehlt. 
Adrenalin stimuliert in gleicher Weise die $\beta_{1}$-Adrenozeptoren des Herzens (ausgeprägte positiv-inotrope und -chronotrope Wirkung) wie auch die $\beta_{2^{-}}$ Adrenozeptoren der Gefäße (Vasodilatation). In höheren Konzentrationen besetzt und aktiviert Adrenalin dann auch die $\alpha$-Adrenozeptoren der Gefäße, weshalb bei niedrigen Konzentrationen eine Abnahme des Gefäßwiderstands, bei hohen eine Zunahme des Gefäßwiderstands mit Blutdruckanstieg resultiert. Adrenalin wird als besonders arrhythmogen angesehen. Die im Schrifttum empfohlene Zurückhaltung beim Einsatz - nur zur Therapie einer ausgeprägten Hypotonie bei gleichzeitig hohem Schlagvolumen - steht in gewissem Widerspruch zu der praktischen Erfahrung, daß mit Adrenalin häufig auch dann noch eine Stabilisierung des Herz-Kreislauf-Systems zu erreichen ist, wenn die anderen genannten Katecholamine keine ausreichende Wirkung mehr zeigen.

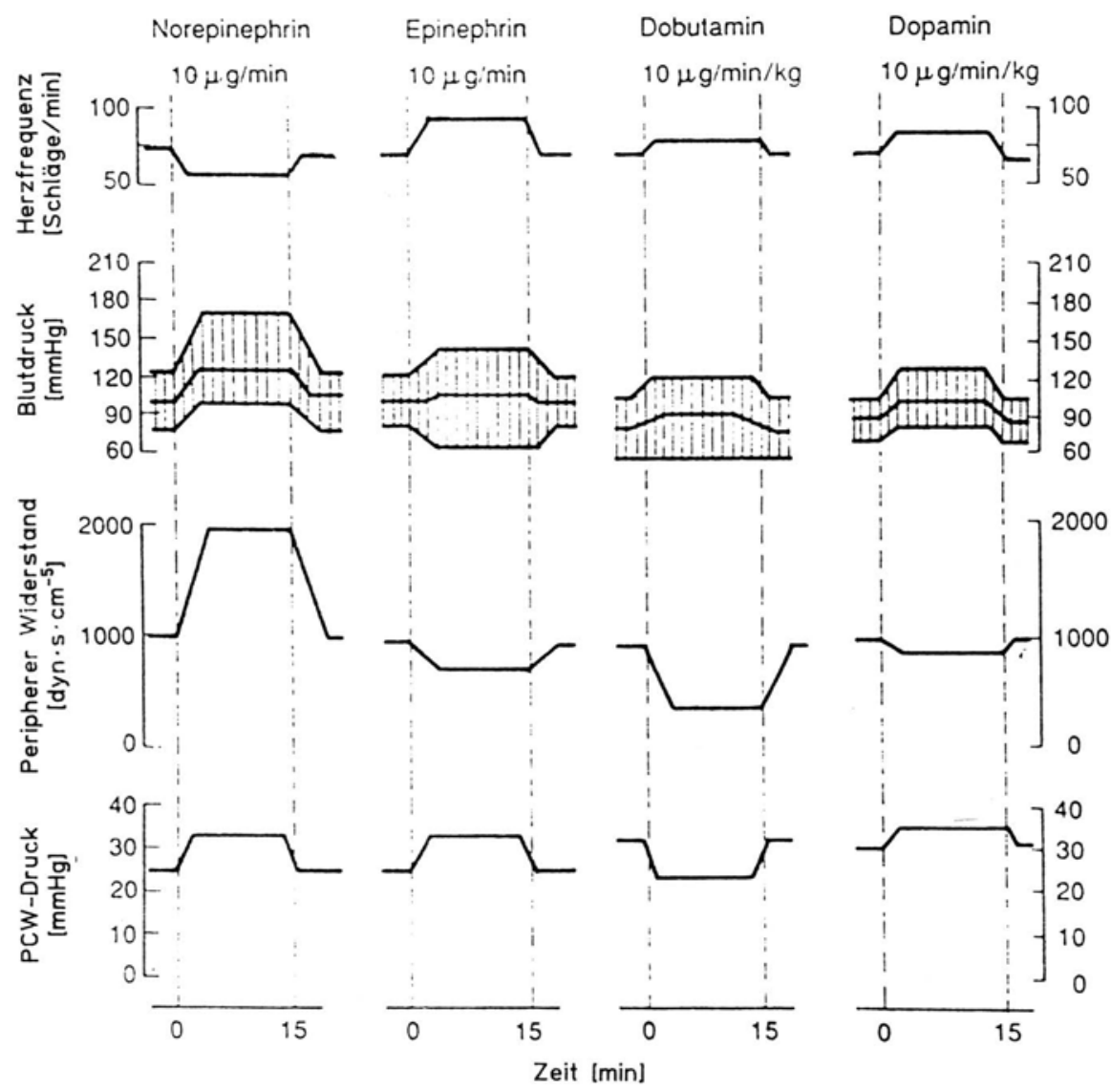

Abb. 10.5. Schematische Darstellung der hämodynamischen Wirkungen der verschiedenen Sympathomimetika („Norepinephrin“ = Noradrenalin; „Epinephrin“ = Adrenalin; „PCW“ = Pulmonalkapillardruck). (Aus Löllgen u. Meuret [9]) 
Der differentialtherapeutische Nutzen dieser unterschiedlichen Rezeptoraffinität wurde von Löllgen u. Meuret [9] anschaulich charakterisiert (Abb. 10.5): Bei vergleichbarer positiv-inotroper Wirkung beeinflussen die 4 aufgeführten Katecholamine Herzfrequenz, Blutdruck und Gefäßwiderstand sowie den linksventrikulär-enddiastolischen Füllungsdruck in unterschiedlicher Weise: Adrenalin wirkt am stärksten, Noradrenalin am wenigsten positiv-chronotrop; v. a. Noradrenalin erhöht den Gefäßwiderstand und damit den Blutdruck; in höheren Konzentrationen als den in Abb. 10.5 aufgeführten tun dies allerdings auch Dopamin und Noradrenalin. Die in Abb. 10.5 gezeigten Katecholaminwirkungen setzen ein intaktes Herz-Kreislauf-System voraus: bei Sepsis und septischem Schock können Desensibilisierungsprozesse und Toxin- und Mediatorschädigungen das Ansprechen auf Katecholamine ganz erheblich beeinträchtigen (s. unten).

Zu erwähnen ist weiterhin Dopexamin, ein Dopaminabkömmling, über dessen Anwendung bisher nur begrenzte Erfahrungen vorliegen. Es stimuliert - schwächer als Dopamin - Dopaminrezeptoren (überwiegend DA-1) und - wesentlich stärker als Dopamin - $\beta_{2}$-Adrenozeptoren; darüber hinaus ist es ein schwacher $\beta_{1^{-}}$ Adrenozeptoragonist, während $\alpha$-Adrenozeptoren durch Dopexamin nicht aktiviert werden. Daraus resultieren ein gesteigerter renaler Blutfluß sowie eine Zunahme des Herzzeitvolumens aufgrund der Nachlastsenkung infolge Vasodilatation. Weiterhin wird eine zusätzliche milde positiv-inotrope Wirkung hervorgerufen: durch Stimulation myokardialer $\beta_{2}$-Adrenozeptoren, durch Potenzierung der Wirkung endogenen Noradrenalins infolge Hemmung der Wiederaufnahme sowie durch eine Aktivierung des Barorezeptorreflexes.

\section{Dopamin und Dobutamin im Vergleich: \\ Füllungsdruck und Wandspannung des linken Ventrikels}

Während Dobutamin den linksventrikulär-enddiastolischen Druck entweder unbeeinflußt läßt oder ihn sogar geringfügig senkt, wird er durch Dopamin meistens etwas gesteigert. Die Ursache für letzteres dürfte ein erhöhter venöser Rückstrom durch eine $\alpha$-Adrenozeptor-vermittelte Venokonstriktion sein ([23], S. 186-196).

Wahrscheinlich als Konsequenz dieser dopamininduzierten Venokonstriktion waren im experimentellen septischen Schock bei Dobutamin größere Flüssigkeitsmengen als bei Dopamin erforderlich, um eine adäquate Volumenauffüllung zu bewirken. Durch die Flüssigkeitssubstitution ließen sich mit Dobutamin Herzzeitvolumen und $\mathrm{O}_{2}$-Transport besser steigern als mit Dopamin.

Über eine günstigere Wirkung des Dobutamins als die des Dopamins auf die rechtsventrikuläre Funktion kritisch Kranker wurde berichtet; desgleichen soll der pulmonale Gasaustausch (Shuntzunahme und/oder Ventilations-PerfusionsMißverhältnis mit resultierender Hypoxämie) durch Dobutamin weniger beeinflußt werden als durch Dopamin. Nach aortokoronarer Bypassoperation waren vergleichbare Dobutaminkonzentrationen wirksamer als Dopamin bezüglich des Herzzeitvolumens und der Inotropieparameter. Die linksventrikuläre Wandspannung stieg unter Dopamin an und blieb unter Dobutamin konstant. Da die Wandspannung eine wesentliche Determinante des myokardialen $\mathrm{O}_{2}$-Verbrauchs 
darstellt, würde Dobutamin v. a. bei koronarkranken Patienten dem Dopamin gegenüber den Vorteil des geringeren myokardialen $\mathrm{O}_{2}$-Verbrauchs bei gleicher oder sogar besserer Wirksamkeit besitzen. Die klinische Relevanz dieses Befundes ist jedoch noch Gegenstand der Diskussion.

\section{Katecholamintherapie und Nierenperfusion}

Beim Einsatz vasokonstriktorischer Katecholamine - v. a. des Noradrenalins und des Dopamins in höherer Dosierung - muß mit einer Einschränkung der Nierenperfusion gerechnet werden.

Zumindest im Hundeversuch konnte die Arbeitsgruppe von Parrillo [12] zeigen, daß die durch Noradrenalin hervorgerufene Widerstandserhöhung der Nierenarterien durch die gleichzeitige Gabe niedrig-dosierten Dopamins verhindert werden kann, mit einer Steigerung des renalen Blutflusses um 40\%. Mit allem Vorbehalt der Übertragbarkeit sprechen diese Befunde dafür, daß die Kombination niedrigdosierten Dopamins - Stimulation der renalen Dopaminrezeptoren mit Noradrenalin die erwünschte blutdrucksteigernde Wirkung des Noradrenalins erbringt, ohne die Nierenperfusion allzu stark zu drosseln. Erste klinische Befunde bei septischem Schock scheinen dieses Konzept - Besserung der Diurese unter einer Kombinationstherapie - zu bestätigen, zumindest bei Patienten mit einem Serumlaktatspiegel von weniger als $20 \mathrm{mg} / \mathrm{dl}$.

\section{Katecholamintherapie und Toleranzentwicklung}

Die positiv-inotrope Wirkung sowohl der endogenen als auch der pharmakologisch applizierten Katecholamine wird vorwiegend durch Stimulation des $\beta_{1^{-}}$ Adrenozeptor-Adenylatzyklasesystems vermittelt (Abb. 10.6). Die Besetzung des $\beta_{1}$-Adrenozeptors in der Herzmuskelzellmembran mit einem Katecholaminmolekül führt über die Aktivierung der Adenylatzyklase zum Anstieg des intrazellulären zyklischen Adenosinmonophosphats (cAMP), über weitere Schritte zu einem Anstieg der zytoplasmatischen Kalziumionenkonzentration und damit schließlich zur positiv-inotropen Wirkung. Stimulatorische $\left(G_{s}\right)$ und inhibitorische $\left(G_{i}\right)$ Guaninnukleotid-bindende Proteine (G-Proteine) regulieren diese rezeptorvermittelte Adenylatzyklasestimulation. Dieses System wird therapeutisch nicht nur beim Einsatz von Katecholaminen, sondern auch bei der Behandlung mit Phosphodiesterasehemmern genutzt.

Bei übermäßiger Stimulation der Adenylatzyklase durch endogene oder exogene Katecholamine, aber auch durch Phosphodiesterasehemmer, kommt es zur Abschwächung der inotropen Katecholaminwirkung am Herzen, hervorgerufen im wesentlichen durch eine Abnahme der Zahl der $\beta_{1}$-Adrenozeptoren auf der Herzmuskelzellmembran und in geringerem Maße auch durch eine Zunahme der $\mathrm{G}_{\mathrm{i}}-$ Proteine. Mit diesen Desensibilisierungsmechanismen versucht die Herzmuskelzelle sich vor einer übermäßigen, deletären Katecholaminaktivierung - im Sinne einer katecholamininduzierten Herzschädigung - zu schützen, unter Inkaufnahme einer Abschwächung der positiv-inotropen Katecholaminwirkung. Durch Züchtung von Kardiomyozyten in Medium mit hohen Katecholaminkon- 


\section{Myokard}

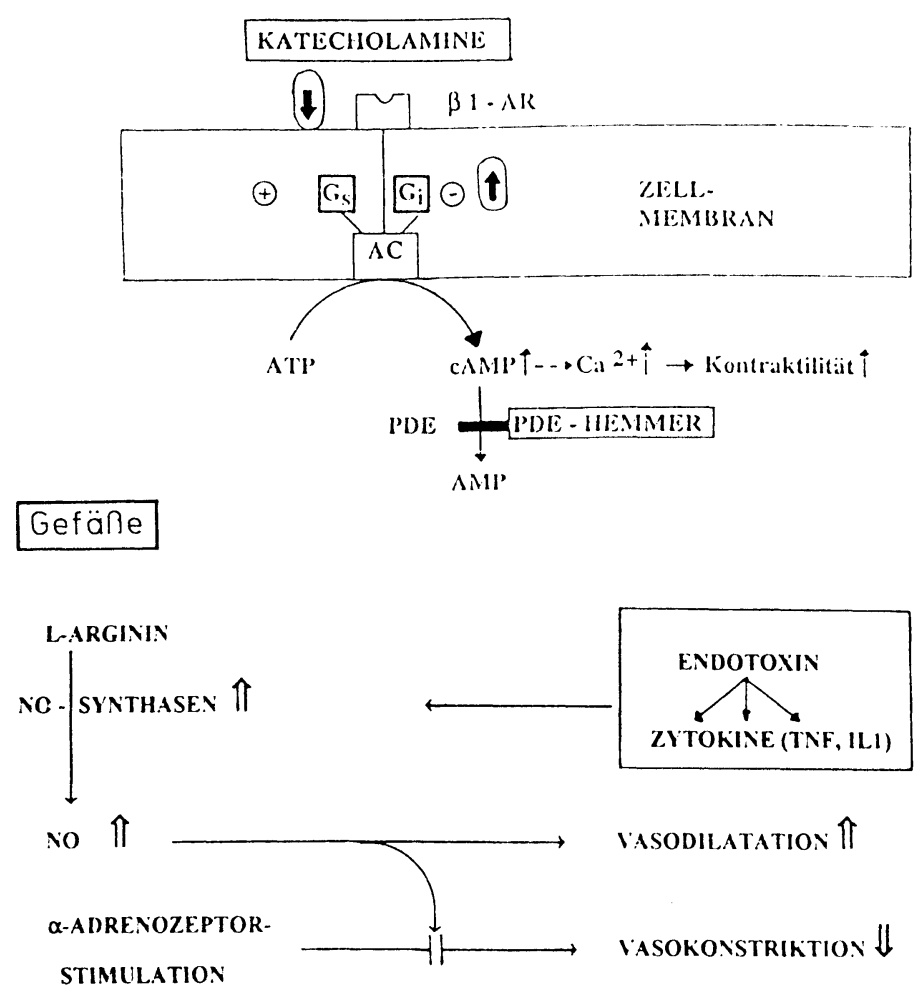

Abb. 10.6. Abschwächung der Katecholaminwirkung an Herz und Gefäßen in der Sepsis. (Erläuterungen s. Text)

zentrationen können diese Desensibilisierungsmechanismen auf zellulärer Ebene untersucht werden (Überblick in [26]): die Wirkungsabschwächung tritt innerhalb von Minuten bis Stunden ein, und sie ist nach Auswaschen des Katecholamins aus dem Züchtungsmedium innerhalb von $24 \mathrm{~h}$ reversibel.

Eine Abnahme der myokardialen $\beta_{1}$-Adrenozeptoren wird bei allen Formen der höhergradigen Herzinsuffizienz gefunden, hervorgerufen durch die hohen extrazellulären Katecholaminspiegel. Auch bei Patienten mit Sepsissyndrom finden sich ca. 10 fach erhöhte Plasma-Noradrenalinspiegel. Demzufolge kann auch bei der akuten septischen Kardiomyopathie die Abnahme der myokardialen $\beta_{1}$-Adrenozeptoren und die daraus resultierende Abschwächung der positivinotropen Wirkung endogener Katecholamine als eine der Ursachen der eingeschränkten Herzfunktion angenommen werden, auch wenn dazu bisher nur tierexperimentelle Befunde vorliegen (Diskussion in [25, 26]).

Beim therapeutischen Einsatz von Katecholaminen läßt sich bei herzinsuffizienten Patienten erwartungsgemäß innerhalb weniger Tage eine Abschwächung 
Tabelle 10.3. Katecholamintoleranzentwicklung $(\overline{\mathrm{x}} \pm \mathrm{SD})$ unter Noradrenalintherapie bei Patienten mit septischem Schock ${ }^{\mathrm{a}}$

\begin{tabular}{llllll}
\hline & Tag 0 & Tag 1 & Tag 2 & Tag 3 & Tag 4 \\
\hline Patientenzahl & 6 & 6 & 6 & 6 & 6 \\
\hline
\end{tabular}

Dosierungen $(\mu \mathrm{g} / \mathrm{min})$ :

Noradrenalin

Adrenalin

Dopamin

$\begin{array}{lllll}0 & 7,4 \pm 3,7 & 14,0 \pm 8,6 & 13,2 \pm 7,0 & 13,8 \pm 7,8 \\ 3,3 \pm 4,7 & 0 & 5,0 \pm 4,2 & 11,0 \pm 12,5 & 4,3 \pm 4,4 \\ 533 \pm 499 & 200 \pm 0 & 200 \pm 0 & 200 \pm 0 & 200 \pm 0\end{array}$

Herz-Kreislauf-Parameter:

Herzindex $\left(1 / \min \cdot \mathrm{m}^{2}\right)$

Arterieller Mitteldruck $(\mathrm{mm} \mathrm{Hg})$

Systemischer Gefäßwiderstand $\left(\mathrm{dyn} \cdot \mathrm{s} \cdot \mathrm{cm}^{-5}\right)$

Linksventrikulärer

Schlagarbeitsindex $\left(\mathrm{g} \cdot \mathrm{m} / \mathrm{m}^{2}\right)$

$$
3,3 \pm 1,0 \quad 3,7 \pm 0,9 \quad 3,6 \pm 1,0
$$$$
68 \pm 16 \quad 65 \pm 5
$$$$
3,9 \pm 1,2
$$$$
67 \pm 8
$$$$
540 \pm 198 \quad 634 \pm 199 \quad 634 \pm 254 \quad 578 \pm 255 \quad 556 \pm 188
$$$$
25 \pm 11 \quad 34 \pm 11 \quad 32 \pm 7 \quad 35 \pm 13 \quad 36 \pm 9
$$

Katecholamindesensibilisierung von Rattenherzmuskelzellen durch Serum (1:1) der oben charakterisierten Patienten (48-h-Inkubation):

$\begin{array}{lll}\beta_{1} \text {-Adrenozeptoren } & 100 \% & 64 \pm 17 \% \\ \text { Adenylatzyklaseaktivität } & 100 \% & 50 \pm 31 \%\end{array}$

a Bei 6 Patienten mit septischem Schock (davon 5 mit kardialen Vorerkrankungen) wurde eine Infusionsbehandlung mit Noradrenalin begonnen und die Auswirkungen auf Herz- und Kreislaufparameter gemessen. Alle Patienten erhielten zusätzlich Dopamin in der angegebenen Dosierung; Adrenalin wurde zusätzlich an Tag 0 - 2 Patienten -, an Tag 2 - 4 Patienten und an den Tagen 3 und $4-3$ Patienten - gegeben.

a) Zur Aufrechterhaltung der hämodynamischen Besserung ist eine Verdoppelung der Noradrenalindosierung notwendig.

b) Das noradrenalinhaltige Patientenserum führt in kultivierten Rattenherzmuskelzellen zur Desensibilisierung des $\beta_{1}$-Adrenozeptor-Adenylatzyklase-Systems: Spontan kontrahierende neonatale Rattenherzmuskelzellen wurden für $48 \mathrm{~h}$ mit noradrenalinfreiem (Tag 0 ) und noradrenalinhaltigem Patientenserum - 1:1 verdünnt - inkubiert. Danach wurde mit Membranpräparationen die Zahl der $\beta_{1}$-Adrenozeptoren mittels Bindung des Radioliganden $\left({ }^{3} \mathrm{H}\right)$ CGP $12177(2 \mathrm{nM})$ und die Isoproterenol $(100 \mu \mathrm{M})$-stimulierte Adenylatzyklaseaktivität im Vergleich zu Kontrollzellen ermittelt, welche zusätzlich mit dem $\beta$-Blocker Timolol $(0,1 \mu \mathrm{M})$ inkubiert worden waren. (Aus Peter et al. [13], S. 62-77)

der Wirkung bis hin zum völligen Wirkungsverlust nachweisen. Auch bei Patienten mit septischem Schock und akuter septischer Kardiomyopathie unterstützen kasuistische Beobachtungen den klinischen Eindruck, daß die durch Gabe von Katecholaminen erreichbare positiv-inotrope Wirkung durch eine zunehmende Katecholamintoleranzentwicklung abgeschwächt wird bzw. mit zunehmender Behandlungsdauer durch eine Steigerung der Katecholamindosierung kompensiert werden muß (Tabelle 10.3): die initial durch Noradrenalin erzielbare Zunahme des linksventrikulären Schlagarbeitsindex bei 6 Patienten mit septischem Schock konnte in den folgenden Tagen nur durch eine Verdoppelung der 
Noradrenalindosis aufrechterhalten werden. Die verwendeten Noradrenalindosierungen können dabei durchaus zur Katecholamindesensibilisierung des Herzens führen: die entsprechenden Patientenseren rufen in RattenherzmuskelZellkulturen innerhalb von $48 \mathrm{~h}$ eine $\beta$-Adrenozeptor-Downregulation um ca. $35 \%$ und eine damit verbundene Hemmung der Adenylatzyklaseaktivität um ca. $50 \%$ hervor (Tabelle 10.3).

Da diese $\beta_{1}$-Adrenozeptor-Adenylatzyklase-Desensibilisierung jedoch alle am myokardialen $\beta_{1}$-Adrenozeptor angreifenden Katecholamine in gleichem Maße betrifft, resultieren daraus keine differentialtherapeutischen Konsequenzen.

Bei chronischer Herzinsuffizienz wird derzeit in kontrollierten Studien versucht, durch $\beta$-Blocker in sehr niedriger Dosierung die downregulierten myokardialen $\beta$-Adrenozeptoren wieder hochzuregulieren und damit die Katecholaminansprechbarkeit wieder zu verbessern. Bei Patienten mit akuter septischer Kardiomyopathie, bei denen ebenfalls von einer Katecholamindesensibilisierung ausgegangen werden muß, liegen bisher keine entsprechenden Erfahrungen vor.

Die myokardialen $\beta_{2}$-Adrenozeptoren zeigen bei herzinsuffizienten Patienten keine Abnahme, trotzdem ist die Ansprechbarkeit auf $\beta_{2}$-Sympathomimetika reduziert. $\alpha_{1}$-Adrenozeptoren stellen im menschlichen Herzen nur etwa $15 \%$ der Gesamtpopulation aller Adrenozeptoren; ob durch ihre Stimulation ein relevanter positiv-inotroper Effekt zu erzielen ist, wird kontrovers diskutiert. Bei höhergradiger Herzinsuffizienz bleibt die Zahl myokardialer $\alpha_{1}$-Adrenozeptoren unverändert bzw. nimmt sogar zu. Dennoch ist die positiv-inotrope Wirkung von $\alpha$-Adrenozeptoragonisten bei terminaler Herzinsuffizienz ebenfalls abgeschwächt.

Auch die Gefäße zeigen im septischen Schock eine Katecholamintoleranz, mit einer abgeschwächten bis fehlenden Vasokonstriktion auf $\alpha$-Adrenozeptoragonisten. In diesem Falle scheint jedoch nicht die im Tierexperiment bei Sepsis und Endotoxinämie gefundene Abnahme der Zahl der Gefäß- $\alpha$-Adrenozeptoren die entscheidende Rolle zu spielen, sondern vielmehr das vermehrt gebildete Stickoxid: durch Hemmstoffe der Stickoxidsynthese läßt sich im Sepsis- und Endotoxintiermodell die stark abgeschwächte vasokonstriktorische Katecholaminwirkung wieder restaurieren (Abb. 10.6; [13], S. 94-103 und S. 104-118). Die Zahl der Gefäß- $\beta_{2}$-Adrenozeptoren ist im Tierexperiment bei Sepsis und Endotoxinämie als nicht verändert beschrieben.

\section{Differentialtherapie mit Katecholaminen}

bei akuter septischer Kardiomyopathie und septischem Schock

Im Gegensatz zum kardiogenen Schock, bei dem die Einschränkung der Pumpleistung des Herzens ganz im Vordergrund steht, mit kompensatorischer Zunahme des systemischen Gefäßwiderstands, dominiert beim septischen Schock primär die toxin- und mediatorbedingte Kreislaufschädigung mit zunehmender Vasodilatation das klinische Bild; bereits in einem frühen - noch normotensiven Stadium kann es aber auch zur ausgeprägten, behandlungspflichtigen Myokarddepression im Sinne der akuten septischen Kardiomyopathie kommen. 


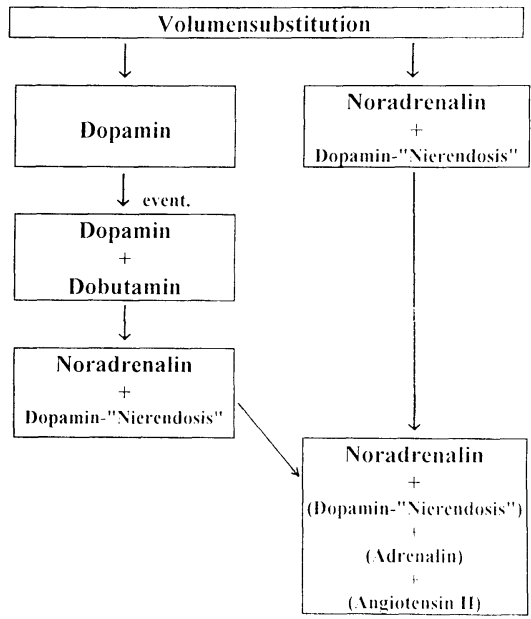

Abb. 10.7. Differentialtherapie mit Katecholaminen bei akuter septischer Kardiomyopathie und septischem Schock. (Erläuterungen s. Text. Das Schema spiegelt das aktuelle Konzept des Autors wider; s. dazu auch [2]; [11], S. 1584-1594; [12]; [20], S. 137-152 und 414-423; [23], S. 186196; Kap.9)

Der erniedrigte Gefäßwiderstand infolge Vasodilatation beim septischen Schock spricht dafür, bei einem notwendig werdenden Einsatz (Abb. 10.7) primär Katecholamine mit vasokonstriktorischer Komponente - Dopamin, Noradrenalin - zu verwenden [25], um einen mittleren Blutdruck von mindestens $60 \mathrm{~mm} \mathrm{Hg}$ aufrechtzuerhalten [12]. Andererseits können auch mit Katecholaminen ohne vasokonstriktorische Komponente - Dobutamin - günstige Ergebnisse erzielt werden, z. B. bei im Vordergrund stehender septischer Kardiomyopathie. Durch die Kombination unterschiedlicher Katecholamine (Abb. 10.7) läßt sich nicht selten therapeutisch mehr erreichen als mit Einzelsubstanzen allein ([2]; [20], S. 414-422; [25]). Selbst bei ausgeprägter Vasodilatation sollte ein reiner $\alpha$-Adrenozeptoragonist - wie z. B. das Phenylephrin - nicht eingesetzt werden, da er durch die Nachlasterhöhung den Herzindex reduziert, ohne die Gewebeperfusion und die $\mathrm{O}_{2}$-Verfügbarkeit im Gewebe zu verbessern ([2]; s. dazu aber [20], S. 414-423).

Katecholamine mit vasokonstriktorischer Komponente haben Vor- und Nachteile. Die bessere Aufrechterhaltung der Gewebeperfusion und die Blutumverteilung aus dem Haut- und Muskelgewebe in die Vitalorgane sind zumindest theoretisch Vorteile der katecholaminbedingten Vasokonstriktion. Potentielle Nachteile spielen v. a. beim septischen Schock keine große Rolle: die Nachlasterhöhung ist in der Regel nicht sehr ausgeprägt, da der Gefäßwiderstand nur selten auf Normalwerte angehoben werden kann. Auch die nachlastbedingte Steigerung des myokardialen $\mathrm{O}_{2}$-Verbrauchs dürfte sich bei dieser Schockform nicht sehr nachteilig auswirken, da die septische Herzschädigung zwar eine Myokarddepression mit erhöhter Ventrikelcompliance beinhaltet, die Koronargefäße jedoch dabei nicht konstringiert, sondern weitgestellt sind ([13], S. 8993).

Bedingt durch die toxin- und mediatorbedingte Schädigung von Herz und Gefäßen und durch die oben beschriebenen Katecholamin-Desensibilisierungsmechanismen sind häufig höhere Katecholamindosen als in Tabelle 10.2 angege- 
ben erforderlich, um überhaupt noch eine positiv-inotrope bzw. vasokonstriktorische Wirkung bei diesen Patienten zu erzielen. Das Vorliegen einer Azidose führt zu einem verminderten Ansprechen des Herzens auf Katecholamine, allerdings erst ab pH $\leq 7,20$ ([23], S. 186-196).

Differentialtherapeutische Aspekte der Katecholamingabe im Hinblick auf eine Optimierung der Gewebeperfusion und des $\mathrm{O}_{2}$-Stoffwechsels im Gewebe werden in Kap. 9 ausführlich abgehandelt. Hier soll nur angefügt werden, daß mit Katecholaminen ohne wie auch mit vasokonstriktorischer Komponente $\mathrm{O}_{2}$ Angebot und $\mathrm{O}_{2}$-Verbrauch gesteigert werden konnten. Sowohl bei postoperativ kritisch Kranken als auch bei septischen Patienten scheint eine günstige Prognose an einen relativ hohen $\mathrm{O}_{2}$-Transport ( $>600$ bzw. $800-1000 \mathrm{ml} / \mathrm{min} / \mathrm{m}^{2}$ ) und $\mathrm{O}_{2}$ Verbrauch ( $>170$ bzw. $180 \mathrm{ml} / \mathrm{min} / \mathrm{m}^{2}$ ) geknüpft zu sein (Zitat 46 in [25]). Damit lägen auch für die Katecholamintherapie anzustrebende quantifizierbare Therapie-Erfolgsparameter vor (s. auch Tabelle 10.1).

\section{Weitere positiv-inotrope und vasoaktive Pharmaka}

\section{Phosphodiesterasehemmstoffe}

Von den Alternativen zu den im wesentlichen bei Patienten mit Sepsis zum Einsatz gelangenden Katecholaminen Dobutamin, Dopamin, Noradrenalin und Adrenalin ist v.a. die Gruppe der positiv-inotrop und vasodilatierend wirkenden Phosphodiesterasehemmer (z. B. Amrinon, Milrinon, Enoximon) zu nennen. Durch Hemmung des Abbaus des cAMP (Abb. 10.6) bewirken sie über einen zusätzlichen, von der $\beta$-Adrenozeptorstimulation unabhängigen Wirkmechanismus einen weiteren Anstieg des zellulären cAMP, was im Herzen einen positivinotropen und in den Gefäßen einen vasodilatierenden Effekt zur Folge hat. Auch mit diesen Substanzen kann eine Verbesserung des $\mathrm{O}_{2}$-Metabolismus erzielt werden (Zitat 24 in [25]). Im Tiermodell (Schweine) des septischen Schocks ließ sich mit dem Phosphodiesterasehemmer Milrinon - ohne zusätzliche Katecholamingabe - zwar das Herzzeitvolumen länger im Normbereich halten, es kam jedoch zu einem stärkeren Blutdruckabfall, die pulmonale Hypertonie wurde nicht wesentlich gesenkt, und die Prognose der Tiere wurde durch Milrinon nicht gebessert, sondern eher verschlimmert. Die schlechte Steuerbarkeit (Halbwertszeit des Enoximons bei herzinsuffizienten Patienten: 3-20 h und eine ausgeprägte Vasodilatation müssen als mögliche Probleme beim Einsatz dieser Substanzen berücksichtigt werden und schränken ihre Anwendung gerade beim septischen Schock doch erheblich ein.

Bei der Kombination eines Katecholamins mit einem Phosphodiesterasehemmer erwartet man sich eine stärkere positiv-inotrope Wirkung als bei der jeweiligen Monotherapie. Bei vorbestehender Vasodilatation (septischer Schock) sollte dann aber eher ein vasokonstriktorisches Katecholamin zum Einsatz gelangen: So wurde über eine günstige Wirkung der Kombination eines Phosphodiesterasehemmers mit Noradrenalin berichtet, mit einer additiven positivinotropen Wirkung ohne Verstärkung der Vasodilatation ([23], S. 186-196). 
Aufgrund der potentiell schwer traktablen Nebenwirkungen und der schlechten Steuerbarkeit bei nicht klar belegter Prognoseverbesserung zählen Phosphodiesterasehemmer derzeit nicht zur Standardtherapie des septischen Schocks. Ihr Einsatz sollte allenfalls beschränkt werden auf Patienten mit einer hypodynamen Form des septischen Schocks, mit stark eingeschränkter Herzfunktion und einem eher hohen systemischen Gefäßwiderstand. Auf eine adäquate vorherige Volumensubstitution ist besonderer Wert zu legen.

\section{Dopexamin}

Durch Dopexamin $(1,0-6,0 \mu \mathrm{g} / \mathrm{kg} / \mathrm{min}) \mathrm{kommt}$ es bei Patienten mit septischem Schock akut zu einem Anstieg des Herzindex (von 4,0 auf 5,0 l// $\mathrm{m}^{2}$ ) und der Herzfrequenz (von 120 auf 130/min) und zu einem Abfall des systemischen Gefäßwiderstandes (von 700 auf $550 \mathrm{dyn} \cdot \mathrm{cm}^{-5} \cdot \mathrm{s}$ ); mittlerer Blutdruck, Pulmonalisdruck und Pulmonalkapillardruck ändern sich nicht signifikant; während einer 48stündigen Infusionsdauer kam es zu einer gewissen Wirkungsabschwächung (Zitat 8 in [25]). Der Einsatz dieser Substanz - soweit derzeit bereits abschätzbar - scheint sich auf Patienten mit hypodynamem septischem Schock auszurichten; eine Überlegenheit gegenüber der Standardkatecholamintherapie ist nicht zu erkennen. Eine selektiv günstige Wirkung auf das Splanchnikusgebiet wird diskutiert (s. Kap. 2).

\section{Angiotensin II}

Im katecholaminrefraktären septischen Schock sind günstige hämodynamische Wirkungen des Angiotensin II (5-20 $\mu \mathrm{g} / \mathrm{min})$ beschrieben worden [22]. Der durch Angiotensin II erzielbare Anstieg des Blutdrucks kann aber mit einer deutlichen Einschränkung des Herzindex einhergehen [2]. Deshalb sollten vor Einleiten der Angiotensinbehandlung die Möglichkeiten der Noradrenalintherapie (vasokonstriktorische bei gleichzeitig positiv-inotroper Wirkung) ausgeschöpft werden.

\section{Vasodilatatoren}

Eine Vasodilatatorentherapie mit Nitroglyzerin- oder Natriumnitroprussidinfusionen ist auf die wenigen Patienten mit hypodynamem septischem Schock trotz adäquater Volumenzufuhr beschränkt, die einen erhöhten systemischen Gefäßwiderstand und einen hohen Pulmonalkapillardruck aufweisen. Darüber hinaus erscheint ein Behandlungsversuch bei Patienten gerechtfertigt, die - bei normalem oder erhöhtem Blutdruck - eine Funktionseinschränkung vitaler Organe infolge Minderperfusion aufweisen. Hier kann durch den Einsatz von Vasodilatatoren versucht werden, die erwünschte Umverteilung der Durchblutung zu erreichen, die sich als Besserung der Organfunktion manifestieren sollte [12]. Kontrovers diskutierte Behandlungsversuche mit den Prostaglandinderivaten $\mathrm{PGE}_{1}$ und PG I $I_{2}$ ([11], S. 1587) betreffen v. a. Patienten mit ARDS. 


\section{Glukagon}

Glukagon wirkt über eine glukagonrezeptorvermittelte Stimulation der Adenylatzyklase am Herzen positiv-inotrop und -chronotrop. Sein Einsatz (1-4-6 mg i.v., danach eine Infusion mit $2-10 \mathrm{mg} / \mathrm{h}$ ) wird eigentlich nur noch empfohlen bei mit $\beta$-Blockern behandelten Patienten mit Hypotonie, bei denen die konventionelle Therapie fehlgeschlagen ist ([2]; [20], S. 414-423; [23], S. 186-196).

\section{Kalzium}

Unter Umgehung desensibilisierter bzw. geschädigter Adrenozeptoren kann über einen sehr beschränkten Zeitraum durch Kalziuminfusionen (20 mmol/h; [4]) ein septischer Schockzustand gebessert werden ([4]; [23], S. 186-196), unter Ausnutzung sowohl der positiv-inotropen als auch der vasokonstriktorischen Kalziumwirkung. Ein Anstieg des ionisierten Serumkalziums limitiert in der Regel dieses Vorgehen nach Stunden [4]. Möglicherweise könnten auch Kalziumagonisten (z. B. Bay K 8644) eine günstige Wirkung im septischen Schock zeigen ([23], S. 186-196); ausreichende klinische Erfahrungen liegen dazu aber nicht vor.

In diesem Zusammenhang erscheint es erwähnenswert, daß die negativinotrope und -chronotrope Wirkung eines im Schock mit erhöhter Aktivität gefundenen, als Peptid identifizierten kardiodepressiven Faktors (CDF) auf eine Blockade des Kalziumeinstroms in die Herzmuskelzelle zurückgeführt werden kann [26].

\section{Digitalis}

Die Wirksamkeit von Digitalis im septischen Schock als positiv-inotropes Agens wurde von Vincent ([23], S. 186-196) als enttäuschend eingestuft. Wie bei anderen Formen der akuten Herzinsuffizienz sind Katecholamine auch im septischen Schock die Inotropika der ersten Wahl, während die Wirkung von Digitalis als bestenfalls geringgradig einzustufen ist. In Abwägung des geringen Nutzens und der potentiell gefährlichen Digitalisüberdosierungen durch Elektrolytstörungen und Nierenfunktionseinschränkungen in der Sepsis sollte die Indikation zur Digitalisierung bei diesen Patienten sehr streng gestellt werden: eine klare Indikation ist nach Ansicht des Autors nur noch bei bestimmten Rhythmusstörungen (z. B. tachykardes Vorhofflimmern, Vorhofflattern) gegeben, unter sorgfältiger Überwachung und ggf. Dosisanpassung.

\section{Diuretika, Hämofiltration und Hämodialyse}

Die Behandlung des prärenalen Nierenversagens bei septischem Schock mit Diuretika (Furosemid, Etacrynsäure), Hämofiltration oder Hämodialyse unterscheidet sich nicht von der Behandlung eines akuten Nierenversagens nichtseptischer Genese. (Siehe dazu auch S. 191-192 und Kap. 8.) 


\section{Rhythmusmonitoring und antiarrhythmische Therapie}

Ein spezifisches Muster an Rhythmusstörungen ist für die akute septische Kardiomyopathie nicht dokumentiert. Dennoch muß mit potentiell malignen Rhythmusstörungen gerechnet werden, wozu Elektrolytentgleisungen, akutes Nierenversagen, Hypo- und Hypervolämie, Katecholamintherapie, kardiale Vorerkrankungen und Fieber beitragen. Ein Patient mit Sepsis und Multiorganversagen sollte kontinuierlich EKG-monitorüberwacht werden. Bei Auftreten von Rhythmusstörungen unterscheidet sich die antiarrhythmische und Defibrillatortherapie nicht grundlegend von der bei nichtseptischen Patienten. Obwohl dem Adrenalin die größte arrhythmogene Wirkung unter den zum Einsatz gelangenden Katecholaminen zugeschrieben wird, können Rhythmusstörungen grundsätzlich unter jedem Katecholamin dosisabhängig auftreten und ein empirisches Umsetzen erforderlich machen.

\section{Oxygenierung, Beatmung und Reduktion der Herzarbeit}

Hypoxämie und eine übermäßige Atemarbeit können zur Verschlimmerung der Herzinsuffizienz beitragen. Die Ausschaltung der Hypoxämie läßt sich durch Wahl der geeigneten Atemunterstützung bzw. Beatmungsform erreichen (s. Kap. 11); eine gemessene arterielle $\mathrm{O}_{2}$-Sättigung von mindestens $90 \%$ und ein arterieller $\mathrm{O}_{2}$-Partialdruck von mindestens $60 \mathrm{~mm} \mathrm{Hg}$ können als Kontrollparameter herangezogen werden [2]. Die aktive Atemarbeit ist am gesamten $\mathrm{O}_{2-}$ Verbrauch mit einem hohen Anteil beteiligt; bei bestimmten Lungen-Thoraxtraumata und Multiorganversagen kann er bis zu 50\% betragen [2]. Bereits bei den ersten Hinweisen auf eine erhöhte Atemarbeit (Tachypnoe mit vermindertem Atemzugvolumen und Hyperventilation) sollte eine entsprechende Atemunterstützung vorgenommen werden (s. Kap. 11), um bei akuter septischer Kardiomyopathie zur Entlastung des Herzens beizutragen. Dazu dienen auch Anxiolyse und Analgesie, Relaxierung zur Einsparung von Sedativa (s. unten) und die Beseitigung von Fieber (Hyperthermie steigert den $\mathrm{O}_{2}$-Verbrauch um $7 \%$ pro ${ }^{\circ} \mathrm{C}$ [2]). Ebenfalls beachtet werden müssen die negativen Auswirkungen der PEEPBeatmung auf die Herzfunktion (Zunahme der rechtsventrikulären Nachlast, Zunahme des rechtsventrikulären Durchmessers und Abnahme der linksventrikulären diastolischen Dehnbarkeit, direkte myokarddepressive Wirkung), ebenso bei CMV und IMV [2].

Die Myokarddepression der akuten septischen Kardiomyopathie kann weiterhin verstärkt werden durch Anästhetika und Barbiturate. Während Benzodiazepine und Opiate für sich keine relevante Myokarddepression hervorrufen - mit Ausnahme von Meperiden -, können sie in Kombination einen additiven negativinotropen Effekt induzieren [2]. 


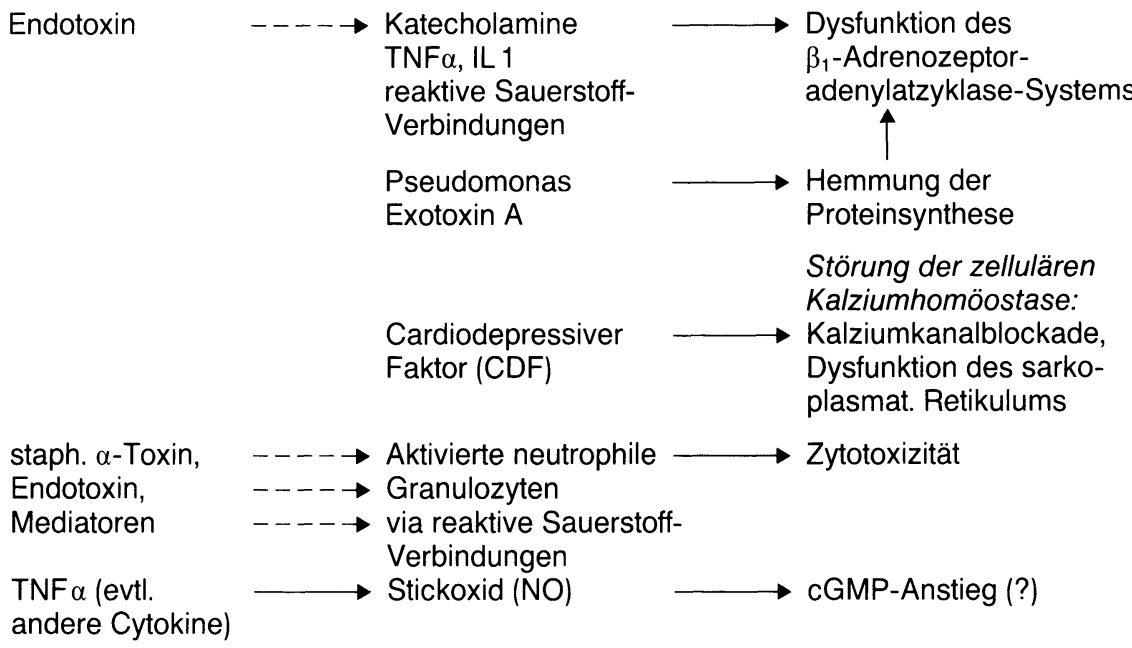

Abb. 10.8. Nachgewiesene „negativ-inotrope Kaskaden“ in Kardiomyozyten, hervorgerufen durch Bakterientoxine und Sepsismediatoren. Das Schema faßt In-vitro-Daten aus Experimenten mit Kardiomyozyten zusammen. Die durchgezogenen Pfeile markieren belegte Reaktionswege; die unterbrochenen Pfeile weisen auf weitere mögliche negativ-inotrope Wirkungen von Bakterientoxinen und Sepsismediatoren hin, die diese durch Stimulation von Mediatorzellen (z. B. neutrophile Granulozyten) und konsekutiver Mediatorfreisetzung (z. B. von TNF $\alpha$ und IL 1) hervorrufen könnten (TNF $\alpha$ Tumornekrosefaktor $\alpha$; IL I Interleukin 1; cGMP zyklisches Guanosinmonophosphat). (Siehe dazu Werdan et al. [26])

\section{Kausale Therapie der akuten septischen Kardiomyopathie}

Bei der akuten septischen Kardiomyopathie muß von einer multifaktoriellen Genese ausgegangen werden; dabei ist der jeweilige Stellenwert der einzelnen Bakterientoxine und Sepsismediatoren noch weitgehend ungeklärt (Abb. 10.8; [26]). Ähnliches muß auch von der Gefäßschädigung in der Sepsis angenommen werden. Hier wird derzeit v. a. dem vasodilatierend wirkenden Stickoxid (NO) ein hoher Stellenwert eingeräumt.

Die kausale Therapie der akuten septischen Kardiomyopathie und der Vasodilatation steckt noch in den Anfängen; sie hat zum Ziel, die zur Herzfunktionseinschränkung und Vasodilatation führende Sepsisschädigungskaskade zu unterbrechen, und zwar durch Neutralisierung, Antagonisierung und Eliminierung der involvierten Bakterientoxine und Sepsismediatoren. Über den Stellenwert dieser Behandlungsversuche kann derzeit noch keine gesicherte Aussage gemacht werden. Von den vielen in Erprobung befindlichen Therapieprinzipien sollen hier überwiegend nur die diskutiert werden, die dem auf der Intensivstation tätigen Arzt bereits heute zur Verfügung stehen.

\section{Endotoxinantikörper}

Während sich ein Endotoxinantikörper („E 5“) im gramnegativen Schock als unwirksam herausgestellt hat (s. Kap. 5), konnte mit dem - zwischenzeitlich aller- 
dings aus dem Handel gezogenen - Endotoxinantikörper HA-1 A (Centoxin, Centocor/Lilly) in einer placebokontrollierten Studie bei der Patientensubgruppe mit nachgewiesener gramnegativer Bakteriämie die Letalität signifikant gesenkt werden, unabhängig davon, ob bei diesen Patienten bereits ein septischer Schock vorlag oder nicht (s. Kap. 5). Gerade Patienten mit gramnegativer Bakteriämie und septischem Schock profitierten von der Endotoxinantikörpergabe noch mehr (Letalitätssenkung von 57\% auf 33\%) als die Patienten ohne septischen Schock (Letalitätssenkung im Gesamtkollektiv aller Patienten mit gramnegativer Bakteriämie von 49 auf $30 \%$ ). Obwohl in dieser Studie nicht explizit erwähnt, ist davon auszugehen, daß die Besserung des septischen Schocks wohl im wesentlichen auf eine Verminderung der Gefäßschädigung mit Anstieg des systemischen Gefäßwiderstands und damit Blutdruckstabilisierung zurückzuführen war. Keine Angaben liegen darüber vor, ob sich auch die Herzfunktion bei diesen Patienten durch die Gabe des Endotoxinantikörpers gebessert hat.

In Form kasuistischer Beobachtungen bei 6 Patienten mit vermuteter gramnegativer Sepsis (nur in einem Fall mit dokumentierter gramnegativer Bakteriämie!) sahen wir nach Gabe von $100 \mathrm{mg}$ Centoxin i.v. innerhalb von 4 Tagen einen Anstieg des systemischen Gefäßwiderstands um knapp 200 E; die akute septische Kardiomyopathie wurde bei diesen Patienten durch den Antikörper nicht gebessert; es kam zu keinem Anstieg des linksventrikulären Schlagarbeitsindex (vgl. Tabelle 10.4).

\section{Tumornekrosefaktor $\alpha$-Antikörper (TNF $\alpha$-AK)}

TNF $\alpha$ wird als einer der wesentlichen Mediatoren der Gefäßschädigung in der Sepsis angesehen, und auch bei der akuten septischen Kardiomyopathie dürfte er ursächlich eine wesentliche Rolle spielen [3, 24]. Placebokontrollierte Sepsistherapiestudien zur Frage einer möglichen Letalitätssenkung durch TNF $\alpha$-AK sind im Gange (s. Kap. 7). Nach Gabe von TNF $\alpha$-AK sahen Vincent et al. [24] innerhalb von $2 \mathrm{~h}$ einen Anstieg des linksventrikulären Schlagarbeitsindex um 19\% (von $26,5 \pm 5,6$ auf $31,5 \pm 10,5 \mathrm{~g} \cdot \mathrm{m} / \mathrm{m}^{2}$ ) im Sinne einer Besserung der bestehenden akuten septischen Kardiomyopathie. Eigene kasuistische Beobachtungen an 9 Patienten mit septischem Schock, die über jeweils 5 Tage mit TNF $\alpha$-AK behandelt worden sind (TNF $\alpha$-AK MAK 195F, Fa. Knoll, Ludwigshafen), dokumentieren einen Anstieg des systemischen Gefäßwiderstands um 226E im Mittel, der vergleichbar der Gabe von Immunglobulin $G$ ist (Tabelle 10.4); die eingeschränkte linksventrikuläre (linksventrikulärer Schlagarbeitsindex) und rechtsventrikuläre (rechtsventrikuläre Auswurffraktion) Herzfunktion bessert sich dagegen bestenfalls geringgradig.

\section{Immunglobuline}

Der Stellenwert der Immunglobuline bei der Behandlung von Sepsis und septischem Schock wird ausführlich in Kap. 5 diskutiert. An dieser Stelle sollen 


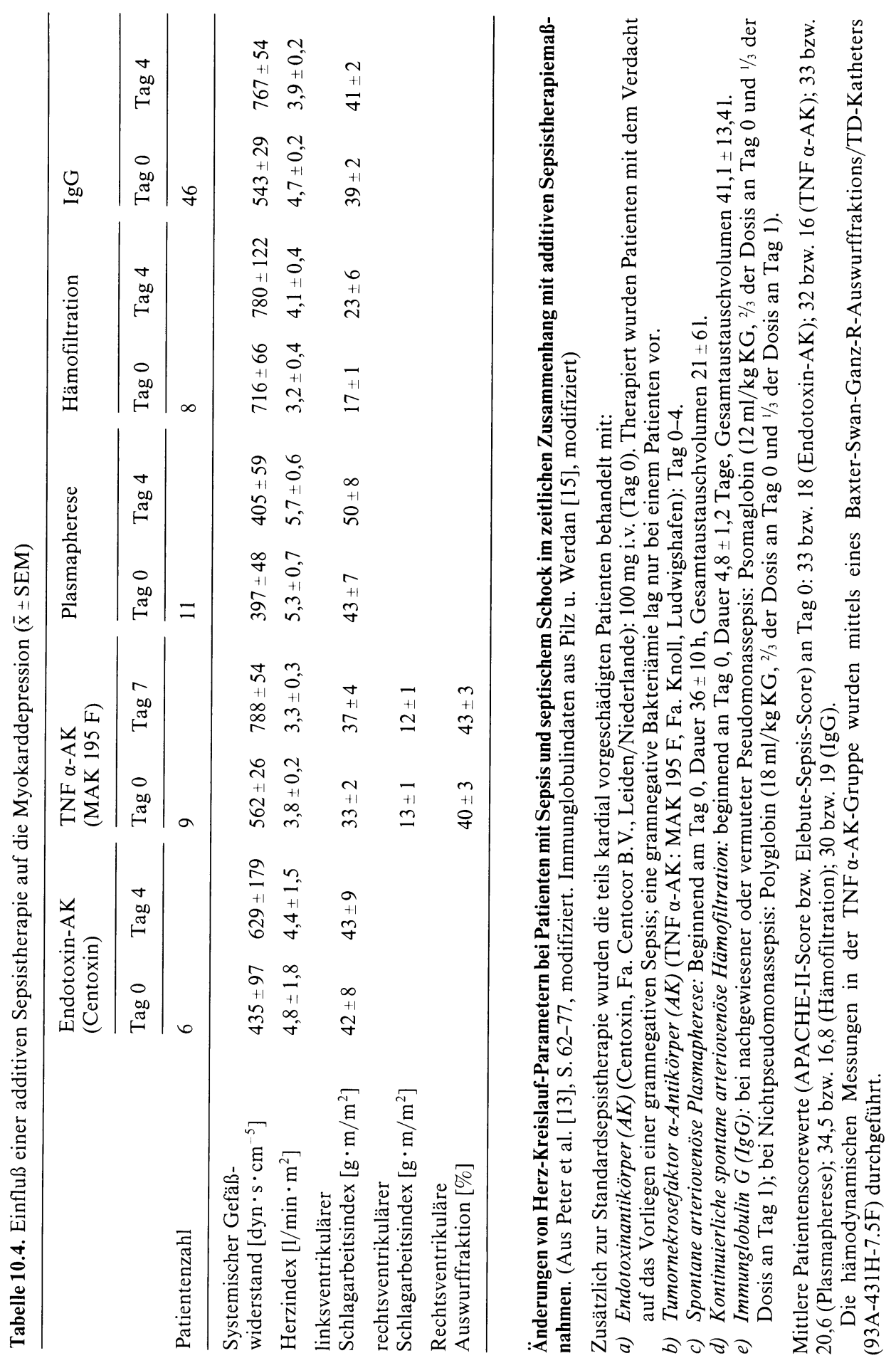


lediglich Aspekte zur Sprache kommen, die sich mit ihrem Einfluß auf die HerzKreislauf-Schädigung bei Sepsis und septischem Schock beziehen:

Bei 62 Patienten mit schwerer postoperativer Sepsis und einem Sepsisscore von $\geq 20$ führte die Gabe eines polyvalenten Immunglobulins G (Sandoglobulin; Sandoz, Basel/Schweiz; 0,4 g/kg KG an den Tagen 0, 1 und 5 gegeben) zu einer Letalitätssenkung von $67 \%$ in der Placebo-(Albumin-)Gruppe, auf $38 \%$ in der Immunglobulin-G-Gruppe (s. Kap. 5). Der Rückgang der Sterblichkeit war dabei ausschließlich auf den Rückgang der Todesfälle infolge septischen Schocks von $33 \%$ auf $7 \%$ zurückzuführen, während der Anteil des Multiorganversagens als Todesursache durch die Immunglobulingabe nicht vermindert wurde (30 vs. $31 \%$ ). Die Befunde sprechen für eine günstige Beeinflussung der septisch bedingten Herz-Kreislauf-Schädigung durch Immunglobulin G.

In die gleiche Richtung weisen die Ergebnisse einer Fallbeobachtungsstudie, bei der Patienten mit Sepsis bzw. septischem Schock mit einem polyvalenten IgGPräparat (Polyglobin N; Troponwerke Biologische Präparate, Köln) bzw. - bei

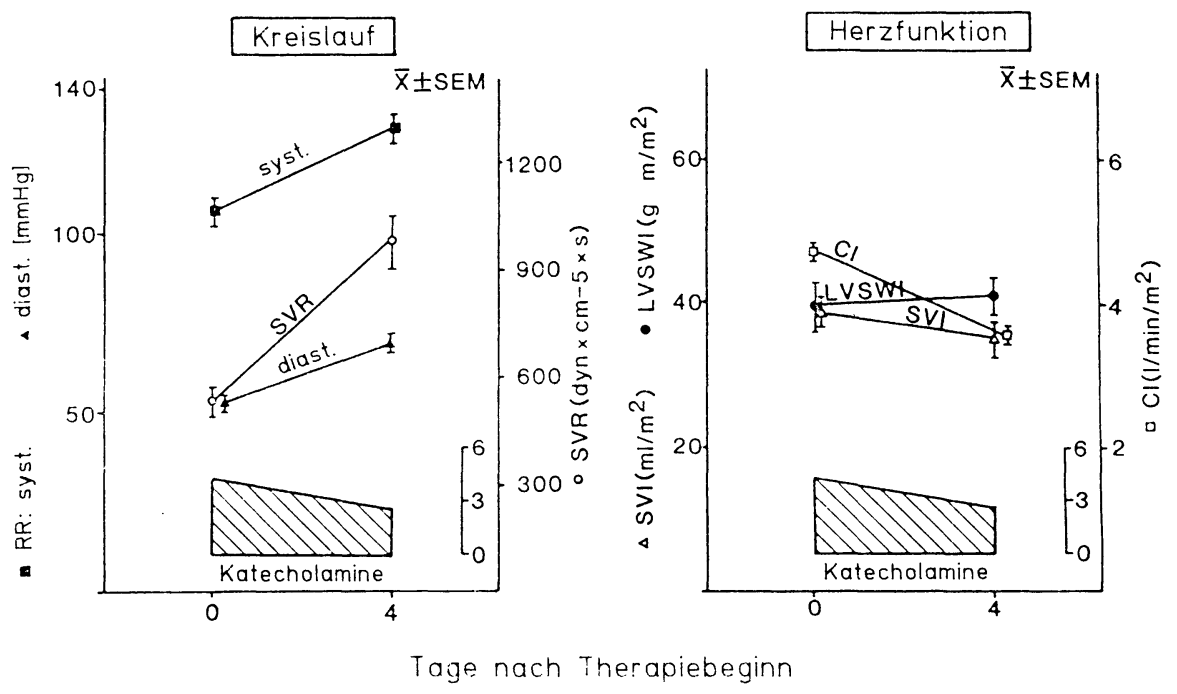

Abb. 10.9. Hämodynamische Änderungen bei Patienten mit septischem Schock in zeitlichem Zusammenhang mit einer additiven Immunglobulingabe. 46 Patienten mit unter Standardtherapie progredientem septischem Schock wurde zusätzlich an 2 Tagen (Tag 0 und 1 in der Abbildung) polyvalentes Immunglobulin $\mathrm{G}$ bzw. Pseudomonasimmunglobulin $\mathrm{G}$ gegeben [15]. Im zeitlichen Zusammenhang mit dieser Immunglobulingabe kam es bei 24 Patienten zu keiner wesentlichen Änderung des erniedrigten systemischen Gefäßwiderstands. Bei 22 Patienten - deren Daten in der Abbildung gezeigt sind - stieg der systemische Gefäßwiderstand ( $S V R$ ) deutlich an (linke Abbildung) und trennte auf diese Weise „hämodynamische Responder“ (Anstieg des SVR $\geq 160 \mathrm{dyn} \cdot \mathrm{cm}^{-5} \cdot \mathrm{s}$ ) mit deutlich besserer Prognose von den „hämodynamischen Non-Respondern“ [15]. Im Gegensatz zur Besserung der Kreislaufsituation konnte bei diesen Patienten keine Besserung der eingeschränkten Herzfunktion (SVI Schlagvolumenindex; LSWI linksventrikulärer Schlagarbeitsindex; $C I$ Herzindex) gemessen werden. Der Abbildung liegen Daten aus [15] zugrunde 
nachgewiesener oder vermuteter Pseudomonassepsis - mit einem Pseudomonasimmunglobulin (Psomaglobin, Troponwerke Biologische Präparate, Köln) behandelt worden sind (Dosierung und zeitlicher Ablauf s. Erläuterungen unter Tabelle 10.4). Wie der Tabelle $10.4 \mathrm{zu}$ entnehmen ist, kommt es dabei in engem zeitlichem Zusammenhang mit der Behandlung zu einem Anstieg des systemischen Gefäßwiderstands um ca. 220 E. Analysiert man die „Gefäßantwort“ auf die Gabe von IgG, so fällt auf, daß etwa die Hälfte aller Patienten mit dem Gefäßwiderstand ansteigt (Abb. 10.9); „Responder“: $\geq 24 \mathrm{~h}$ anhaltender Anstieg des systemischen Gefäßwiderstands um mindestens $160 \mathrm{E}$ innerhalb von 4 Tagen nach Beginn der Immunglobulinbehandlung [15], während die andere Hälfte der Patienten keine Besserung des erniedrigten Gefäßwiderstands zeigt („Non-Responder“). Parallel zum Anstieg des systemischen Gefäßwiderstands fällt der Herzindex ab (Tabelle 10.4, Abb. 10.9). Dieser Abfall ist nicht als eine Zunahme der Herzschädigung unter Immunglobulingabe $\mathrm{zu}$ deuten, sondern er resultiert aus der inversen Abhängigkeit des Herzindex vom systemischen Gefäßwiderstand (s. Abb. 10.2 und Kap. 2: Abb. 2.5).

Die hämodynamischen „Immunglobulinresponder“ haben eine signifikant bessere Prognose als die „Non-Responder“ (Letalität $27 \%$ vs. $77 \%$ nach [15]), und die Besserung der Kreislauffunktion korreliert auch mit einer Besserung des Multiorganversagens, was sich anhand des Abfalles des APACHE-II-Scores bei diesen Patienten dokumentieren läßt [15]. Auch eine Abnahme des in der Sepsis erhöhten Skelettmuskel- $\mathrm{pO}_{2}$ durch Psomaglobin wurde beschrieben [1], ein Befund, der als eine Steigerung der gestörten $\mathrm{O}_{2}$-Verwertung im Skelettmuskel durch das Immunglobulin gedeutet werden könnte.

Die gleichen „Responder“, die auf die Gabe von IgG mit einem Anstieg des Gefäßwiderstands und des Blutdrucks reagieren (Abb. 10.9, Tabelle 10.4), erfahren jedoch keine Besserung der ebenfalls vorhandenen akuten septischen Kardiomyopathie: der erniedrigte linksventrikuläre Schlagarbeitsindex zeigt keinen relevanten Anstieg. Dies könnte darauf hindeuten, daß der Schädigung des Kreislaufs und des Herzens in der Sepsis unterschiedliche Schädigungsmechanismen zugrunde liegen, die sich unabhängig voneinander bessern können bzw. unterschiedlich beeinflußt werden können.

\section{Extrakorporale Therapieverfahren: Hämofiltration und Plasmapherese}

Extrakorporale Therapieverfahren werden bei Sepsis und Multiorganversagen nicht nur zur Behandlung des akuten Nierenversagens eingesetzt, sondern auch in der Vorstellung, schädliche Bakterientoxine und Sepsismediatoren zu eliminieren. Im Tierexperiment lassen sich damit bei Sepsis sowohl eine Besserung der Kreislaufschädigung (Anstieg des systemischen Gefäßwiderstands) bei Schweinen (Zitat 60 in [7]) als auch eine Besserung der linksventrikulären Dysfunktion bei Hunden (Zitat 18 in [7]) nachweisen.

Zwar ist bei Patienten mit septischem Schock über eine Besserung der Herzfunktion durch Hämofiltration berichtet worden (Zitat 19 in [7]); es fehlen 
allerdings bisher noch kontrollierte Studien hinsichtlich des Einflusses einer Hämofiltration bzw. Plasmapherese auf die Herz-Kreislauf-Funktion.

Bei postoperativem Nierenversagen - nicht nur septischer Genese - korrelierte die Menge des täglichen Ultrafiltrates ( $>151 /$ Tag vs. $<151 /$ Tag) signifikant mit der Überlebensrate der Patienten; ob dies v.a. auf eine Besserung der HerzKreislauf-Schädigung zurückzuführen ist, läßt sich dabei nicht ersehen (s. Kap. 8). Durch Hämoperfusion mit einer Polymyxinsäule zur Endotoxinelimination ließ sich bei 42 Sepsispatienten die Endotoxinämie signifikant senken (von $85 \mathrm{pg} / \mathrm{ml}$ auf $28 \mathrm{pg} / \mathrm{ml}$ innerhalb eines Tages nach Therapiebeginn); die Behandlung besserte auch die Herz-Kreislauf-Funktion (Anstieg des systemischen Gefäßwiderstands und des Herzindex sowie des $\mathrm{O}_{2}$-Verbrauchs) dieser Patienten [8]. Eigene kasuistische Erfahrungen mit Plasmapherese belegen bei 4 von 10 Patienten mit septischem Schock einen relevanten Anstieg des systemischen Gefäßwiderstands innerhalb von 24-48 h nach Beginn der Plasmapheresebehandlung [7]; bezogen auf das Gesamtkollektiv von jetzt 11 Patienten findet sich jedoch keine eindeutige günstige Wirkung auf Kreislauf (systemischer Gefäßwiderstand) und Herzfunktion (Herzindex, linksventrikulärer Schlagarbeitsindex; Tabelle 10.4). Auch in engem zeitlichen Zusammenhang mit der Hämofiltration konnten wir bei 8 Patienten mit septischem Schock keine wesentliche Besserung der Herz- und Kreislauffunktion erkennen (Tabelle 10.4).

\section{Kortikoide}

In 3 großen kontrollierten Sepsisstudien konnte die hochdosierte Gabe von Glukokortikoiden die Letalität nicht senken (Zitate 81-83 in [13], S. 104-118). Bemerkenswert ist jedoch, daß in einer dieser Studien (Zitat 81) mit Patienten im refraktären septischen Schock die Schocksymptomatik bei 11 der 43 mit Glukokortikoid behandelten Patienten innerhalb von $24 \mathrm{~h}$ beseitigt werden konnte, dagegen bei keinem der Patienten der Kontrollgruppe.

Unter der Vorstellung, daß eine Subgruppe von Patienten im septischen Schock ein relatives Kortikoiddefizit entwickelt, wurden Patienten im septischen Schock mit hochphysiologischen Dosen von Hydrocortison (100-mg-Bolus, kontinuierliche Infusion von $10 \mathrm{mg} / \mathrm{h}$ ) therapiert ([13], S. 104-118). Bei insgesamt 20 der 30 behandelten Patienten konnte innerhalb von $48 \mathrm{~h}$ die Katecholamindosierung um mehr als $60 \%$ reduziert werden; diese "Responder" - mit niedrigeren Cortisolspiegeln als „Non-Responder" - hatten eine niedrigere Letalität.

\section{Naloxon}

Die im septischen Schock erhöhten Plasmaendorphinspiegel (Zitat 9 in [21]) werden für die Hypotension mitverantwortlich gemacht. Eine vorübergehende Anhebung des Blutdrucks läßt sich gelegentlich durch die hochdosierte Gabe des Opiatantagonisten Naloxon erzielen ([20], S.401-413; [21]). Bei fraglichem 
Therapieerfolg kann dieses Vorgehen wegen der z. T. schwerwiegenden Nebenwirkungen [21] derzeit nicht empfohlen werden.

\section{Zyklooxygenasehemmer}

Trotz vielversprechender tierexperimenteller Befunde (Diskussion in [13], S. 104118) mit Zyklooxygenasehemmern wie Indomethacin und Ibuprofen konnten in einer kürzlich publizierten, allerdings methodisch kritisierten klinischen Studie keine vorteilhaften Wirkungen von Ibuprofen auf die Hämodynamik (Herzzeitvolumen, systemischer Gefäßwiderstand) bei schwerer Sepsis nachgewiesen werden [6].

\section{Pentoxifyllin}

Der Phosphodiesterasehemmer Pentoxifyllin hemmt über einen.Anstieg des intrazellulären cAMP die Transkription von TNFmRNS in Makrophagen/ Monozyten. Die in Sepsistiermodellen gefundenen günstigen Effekte auf die Schädigung von Herz/Kreislauf - Abschwächung des Blutdruckabfalls und der Abnahme des Herzzeitvolumens (Diskussion in [11], S. 1587; [13], S. 104-118) werden derzeit in klinischen Studien überprüft.

\section{Hemmung der Stickoxidproduktion}

Die therapierefraktäre Hypotension im Rahmen des septischen Schocks wird zumindest zum überwiegenden Teil auf eine endotoxin- und zytokininduzierte Überproduktion des vasodilatatorisch wirksamen Stickoxids (NO) in Endothelund Gefäßmuskelzellen zurückgeführt, ebenso wie das verminderte Ansprechen der Gefäße auf $\alpha$-Sympathomimetika ([13], S. 94-103). Auch die direkten negativinotropen Wirkungen von Zytokinen (Tumornekrosefaktor $\alpha$, Interleukin 2, Interleukin 6) sind - zumindest im Hamstermyokard - durch Stimulation einer Stickoxidsynthase mit Produktion von Stickoxid vermittelt [5].

Patienten mit Sepsis haben erhöhte Plasmaspiegel der stabilen Abbauprodukte des Stickoxids. Erste klinische Erfahrungen [14] überzeugen bisher jedoch noch nicht in ausreichendem Maße, daß man mit den derzeit verfügbaren Hemmstoffen der Stickoxidsynthase [z. B. $\mathrm{N}^{\mathrm{G}}$-monomethyl-L-arginine (L-NMMA)] gefahrlos eine günstige Wirkung auf die Herz-Kreislauf-Schädigung in der Sepsis ausüben kann ([10]; [13], S. 94-103 und S. 104-118; [27]).

\section{Kausale Therapie der akuten septischen Kardiomyopathie: gegenwärtiger Stand}

Trotz der Aufklärung zahlreicher Toxin- und Zytokinwirkungen auf Herz und Kreislauf und der Entwicklung von Toxin- und Mediatorantikörpern müssen 
aufgrund der vorliegenden Studienergebnisse die kausalen Therapiemöglichkeiten derzeit noch als sehr limitiert eingestuft werden. Die septische Kreislaufschädigung mit Blutdruckabfall infolge der ausgeprägten Vasodilatation scheint dabei noch besser angehbar als die akute septische Kardiomyopathie (Abb. 10.9; Tabelle 10.4).

Die akute septische Kardiomyopathie wird besonders augenscheinlich, wenn die Herzfunktionsparameter in Abhängigkeit vom systemischen Gefäßwiderstand beurteilt werden (Abb. 10.2). Die Ergebnisse einer kausalen Behandung der Herzinsuffizienz in der Sepsis stehen derzeit noch hinter denen der symptomatischen Therapie mit Katecholaminen zurück (vgl. Tabellen 10.3 und 10.4).

Bei kritischer Wertung der vorliegenden Untersuchungsergebnisse muß betont werden, daß viele der aufgeführten Daten aus jeweils einer einzelnen Studie stammen bzw. bisher nur von jeweils einer einzelnen Untersuchergruppe beschrieben worden sind und somit noch ihrer Bestätigung und Absicherung bedürfen.

\section{Spezielle Aspekte bei der Behandlung der rechtsventrikulären Dysfunktion bei akuter septischer Kardiomyopathie ([13], S. 89-93)}

Obwohl die Linksherzinsuffizienz klinisch mehr im Vordergrund steht, besitzt auch die Einschränkung der rechtsventrikulären Pumpfunktion eine große prognostische Bedeutung. Sie resultiert aus der dokumentierten systolischen und auch diastolischen (Linksverschiebung der Druck-Volumen-Kurven) Funktionsstörung. Neben einer dominanten Myokarddepression werden v. a. die pulmonale Hypertonie bei ARDS für das primär rechtskardiale Pumpversagen verantwortlich gemacht, während koronare Perfusionsstörungen eher die Ausnahme darstellen. Rechtsventrikuläre Dilatation und Abnahme der rechtsventrikulären Auswurffraktion sind der Messung mit einem speziellen Rechtsherzkatheter (s. Tabelle 10.4) zugänglich; sie führen sekundär zu einer Abnahme des linksventrikulären Füllungsdrucks und damit auch zur linksventrikulären Pumpfunktionseinschränkung. Die rechtsventrikuläre Dysfunktion kann sowohl isoliert als auch in Kombination mit einer Einschränkung der linksventrikulären Auswurffraktion vorkommen.

Auch bei der Behandlung der rechtsventrikulären Dysfunktion ist der erste Schritt die Volumensubstitution zum Ausgleich einer Hypovolämie, unter Messung der rechts- und linksventrikulären Füllungsdrücke und ggf. auch der rechtsventrikulären Volumina. Das Ausmaß der Volumensubstitution wird gerade bei im Vordergrund stehender rechtsventrikulärer Dysfunktion kontrovers diskutiert: Bei einer kleinen Patientengruppe mit rechtsventrikulärer Dilatation und niedrigem Koronarperfusionsdruck läßt sich durch Volumensubstitution allein das Herzzeitvolumen nicht ausreichend steigern; bei diesen Patienten ist der frühzeitige Einsatz von vasokonstriktorischen Katecholaminen zur Anhebung des Koronarperfusionsdruckes erforderlich.

Persistieren nach der Volumenkorrektur Hypotonie und Schock, so empfiehlt Dhainaut ([13], S. 89-93) als nächsten Schritt den differentialtherapeutischen Einsatz von Katecholaminen: bei normaler rechtsventrikulärer Auswurffraktion 
Dopamin, bei erniedrigter rechtsventrikulärer Auswurffraktion die Kombination von Dopamin und Dobutamin. Insgesamt ist jedoch die Diskussion um das „ideale“ Katecholaminregime bei primär rechtsventrikulärer septischer Herzschädigung noch in vollem Gange (Zitate 44 und 54 in [25]); die Möglichkeit zur detaillierten Quantifizierung des Therapieerfolgs wird im Einzelfall sicherlich hilfreich sein.

\section{Akute septische Kardiomyopathie und kardiale Vorerkrankungen}

Kardiale Vorerkrankungen können die Myokarddepression der akuten septischen Kardiomyopathie überlagern und aggravieren: Stenose-/Insuffizienz- oder Shuntvitium, dilatative oder hypertrophische Kardiomyopathie, dekompensiertes Hochdruckherz, Endo-/Myo-/Perikarditis im Rahmen der akuten infektiösen Erkrankung. Quantitativ die größte Rolle dürfte das Zusammentreffen einer septischen Herzschädigung mit einer koronaren Herzerkrankung spielen ([18]; [20], S. 104-123) - mit Überlagerung der septisch bedingten Myokarddepression mit der Myokardischämie im Rahmen der koronaren Herzkrankheit. Das bei Vasodilatation gesteigerte Herzzeitvolumen erfordert einen höheren myokardialen $\mathrm{O}_{2}$-Verbrauch, der bei fixierten Koronarstenosen zur Verstärkung einer regionalen Myokardischämie führen kann. Eine laufende antianginöse Therapie mit $\beta$-Blockern, Nitraten und Kalziumantagonisten kann wiederum die labile Herz-Kreislauf-Situation des Septikers verschlechtern. Auf die erhöhte Volumenempfindlichkeit dieser „steifen Ventrikel“ wurde bereits hingewiesen, ebenso auf die Bevorzugung des Dobutamins gegenüber dem Dopamin (s. S. 177-178). Differentialdiagnostische Probleme mit differentialtherapeutischen Konsequenzen kann das Zusammentreffen von kardiogenem und septischem Schock bereiten, z. B. das Auftreten einer Sepsis bei einem beatmeten Patienten mit kardiogenem Schock nach Myokardinfarkt. Invasive Parameter (Herzindex und systemischer Gefäßwiderstand) liefern zwar hier rasch eine Klärung über das dominierende Schockgeschehen; dagegen sind nichtinvasive Einzelparameter wie Fieber, Leukozytose und Blutlaktat wenig aussagekräftig [16]. Hier sind Scoresysteme zur Differenzierung hilfreich: so unterscheiden der APACHE-II- und der Sepsisscore nach Elebute u. Stoner mit ausreichender Sensitivität und Spezifität den kardiogenen vom septischen Schock: APACHE-II-Score: 20,3 $\pm 8,4$ (SD) (kardiogener Schock) vs. 29,3 $\pm 6,6$ (septischer Schock); Sepsisscore: 11,0 $\pm 5,8$ vs. $19,0 \pm 5,9[16]$.

\section{Therapie der Herzinsuffizienz bei primär abakteriellem Multiorganversagen}

Die Mehrzahl der Daten zur Pathophysiologie und Therapie der Herzinsuffizienz bei Sepsis und Multiorganversagen wurde bei Patienten mit bakterieller Sepsis und septischem Schock erhoben. Die gemeinsame Mediator-/Zytokinendstrecke macht es verständlich, daß die akute septische Kardiomyopathie sich bei 
gramnegativer und grampositiver Infektion sehr ähnlich darstellt; demzufolge sind die in diesem Artikel beschriebenen therapeutischen Maßnahmen auch nicht unterschiedlich, von wenigen Ausnahmen - wie z. B. dem Einsatz von Endotoxinantikörpern speziell bei gramnegativer Sepsis - einmal abgesehen. Sehr viel weniger bekannt ist über die Herz-Kreislauf-Schädigung bei systemischen Virusund Pilzerkrankungen; die symptomatische Therapie der Herzinsuffizienz bei diesen Erkrankungen unterscheidet sich derzeit nicht von der bei bakterieller Sepsis.

Bei den Patienten mit Multiorganversagen ohne zugrundeliegende Infektion repräsentieren Traumapatienten eine relevante Gruppe [2]. Auch bei diesen Patienten mit SIRS kann eine hyperdyname Herz-Kreislauf-Reaktion nachgewiesen werden; die Myokardeinschränkung scheint jedoch geringer als bei vergleichbar hyperzirkulatorischen Sepsispatienten zu sein [19]; eine notwendige symptomatische Therapie der Herzinsuffizienz ist vergleichbar.

Bei SIRS-Patienten mit hypovolämischem Schock kommt es nach Blutungen, nach Verbrennungen mit Plasmaverlust oder bei ausgeprägter Vasodilatation (anaphylaktischer Schock) zu einem starken Abfall des effektiven intravasalen Blutvolumens und damit zur Vorlastabnahme und zur Absenkung des Herzzeitvolumens. Neben der sicherlich dominanten Myokardhypoxie infolge des erniedrigten koronaren Perfusionsdruckes werden bei diesen Patienten auch noch weitere zur Myokarddepression führende, in ihrem Stellenwert aber noch zu bestimmende Mechanismen angenommen, wie z. B. eine Katecholamindesensibilisierung infolge erhöhter Plasmakatecholaminspiegel und das Auftreten myokarddepressiver Faktoren ([13], S. 62-77). Die symptomatische Therapie der Herzinsuffizienz dieser Patienten folgt ebenfalls den oben S. 172-187 gegebenen Vorschlägen.

\section{Literatur}

1. Boekstegers P, Weidenhöfer S, Werdan K (1991) Continuous measurement of peripheral tissue oxygenation in patients with sepsis: effect of systemic Pseudomonasimmunoglobulin treatment on mean skeletal muscle. Circ Shock 34 [Abstracts]:75

2. Conrad SA, Finkelstein JL, Madden MR, Burk J, Goodwin CW (1990) Cardiovascular dysfunction in multiple organ failure. In: Deitch EA (ed) Multiple organ failure pathophysiology and basic concepts of therapy. Thieme, New York, pp 172-191

3. De Meules JE, Pigula FA, Mueller M, Raymond SJ, Gamelli RL (1992) Tumor necrosis factor and cardiac function. J Trauma 32:686-692

4. Erdmann E, Reuschel-Janetschek E (1989) Hämodynamik in der Sepsis und im septischen Schock. Intensivmedizin 26 [Suppl 1]:16-21

5. Finkel MS, Oddis CV, Jacob TD, Watkins SC, Hattler BG, Simmons RL (1992) Negative inotropic effects of cytokines on the heart mediated by nitric oxide. Science 257:387-389

6. Haupt MT, Jastremski MS, Clemmer TP, Metz CA, Goris GB, the Ibuprofen Study Group (1991) Effect of ibuprofen in patients with severe sepsis; a randomized, doubleblind, multicenter study. Crit Care Med 19:1339-1347

7. Kääb S, Pilz G, Appel R, Samtleben W, Gurland HJ, Werdan K (1992) Extrakorporale Therapieverfahren: Möglichkeiten und klinische Relevanz bei Patienten mit septischem Multiorganversagen. In: Deutsch E, Kleinberger G, Lenz K, Ritz R, Schneeweiß B, 
Schuster HP, Simbruner G, Slany J (Hrsg) Multiorganversagen. Springer, Wien New York (Intensivmedizinisches Seminar, Bd 4, S 139-153)

8. Kodama M, Aoki H, Tani T, Hanasawa K (1992) Hemoperfusion using polymyxin B immobilized fiber column for removal of endotoxin. Abstract-Band: Second Conference of the International Endotoxin Society, Wien, 17.-20. August 1992, Abstr 38, S 38

9. Löllgen H, Meuret GH (1989) Empfehlungen zur Therapie mit Katecholaminen in der Notfallmedizin. In: Vogel F (Hrsg) Differentialtherapie mit Katecholaminen Pathophysiologische und klinische Aspekte. Thieme, Stuttgart (Intensivmedizin Notfallmedizin Anästhesiologie, Bd 70, S 11-13)

10. Nava E, Palmer RMJ, Moncada S (1991) Inhibition of nitric oxide synthesis in septic shock: how much is beneficial? Lancet 338:1555-1557

11. Niemer M, Nemes C, Lundsgaard-Hansen P, Blauhut B (1992) Datenbuch Intensivmedizin. Fischer, Stuttgart Jena New York (Datenbuch Anästhesiologie und Intensivmedizin, Bd 2)

12. Parrillo JE (1989) Septic shock in humans: clinical evaluation, pathogenesis, and therapeutic approach. In: Shoemaker WC, Ayres S, Grenvik A, Holbrook PR, Thompson WL (eds) Textbook of critical care, 2nd edn. Saunders, Philadelphia, pp 1006-1024

13. Peter K, Lawin P, Bein T (Hrsg) (1992) Intensivmedizin 1992. Thieme, Stuttgart New York (Intensivmedizin Notfallmedizin Anästhesiologie, Bd 81)

14. Petros A, Bennett D, Vallance P (1991) Effect of nitric oxide synthase inhibitors on hypotension in patients with septic shock. Lancet 338:1557-1558

15. Pilz G, Werdan K (1990) Cardiovascular parameters and scoring systems in the evaluation of response to therapy in sepsis and septic shock. Infection 18:253-262

16. Pilz G, Stäblein A, Reuschel-Janetschek E, Autenrieth G, Werdan K (1989) The use of scoring systems in patients with cardiogenic and septic shock. In: Schlag G, Redl H (eds) Second Vienna Shock Forum. Liss, New York (Progress in clinical and biological research, Vol 308, pp 625-631)

17. Rackow EC, Kaufmann BS, Falk JL, Astiz ME, Weil MH (1987) Hemodynamic response to fluid repletion in patients with septic shock: evidence for early depression of cardiac performance. Circ Shock 22:11-22

18. Raper RF, Sibbald WJ (1988) The effects of coronary artery disease on cardiac function in nonhypotensive sepsis. Chest 94:507

19. Raper R, Sibbald WJ, Driedger AA, Gerow K (1989) Relative myocardial depression in normotensive sepsis. J Crit Care 4:9-18

20. Reinhart K, Eyrich K (Hrsg) (1989) Sepsis. Springer, Berlin Heidelberg New York Tokyo

21. Rock P, Silverman H, Plump D, Kecala Z, Smith P, Michael JR, Summer W (1985) Efficacy and safety of naloxone in septic shock. Crit Care Med 13:28-33

22. Thomas VL, Nielsen MS (1991) Administration of angiotensin II in refractory septic shock. Crit Care Med 19:1084-1086

23. Vincent JL, Thijs LG (eds) (1987) Septic shock - European view. Update in intensive care and emergency medicine 4. Springer, Berlin Heidelberg New York Tokyo

24. Vincent J-L, Bakker J, Marecaux G, Schandene L, Kahn RJ, Dupont E (1992) Administration of anti-TNF antibody improves left ventricular function in septic shock patients. Results of a pilot study. Chest 101:810-815

25. Werdan K, Reithmann C (1992) Differentialtherapie mit Katecholaminen. In: Schüttler J, Schwilden H, Lauven PM (Hrsg) Klinische Pharmakologie und rationale Arzneimitteltherapie. Thieme, Stuttgart New York (Intensivmedizin Notfallmedizin Anästhesiologie Bd 80, S 133-143

26. Werdan K, Boekstegers P, Müller U, Pfeifer A, Pilz G, Reithmann C, Hallström S, Koidl B, Schuster HP, Schlag G (1991) Akute septische Kardiomyopathie: Bestandteil des Multiorganversagens in der Sepsis? Med Klin 86:526-534

27. Wright CE, Rees DD, Moncada S (1992) Protective and pathological roles of nitric oxide in endotoxin shock. Cardiovasc Res 26: 48-57 